NASA/CR-2000-210103

ICASE Report No. 2000-17

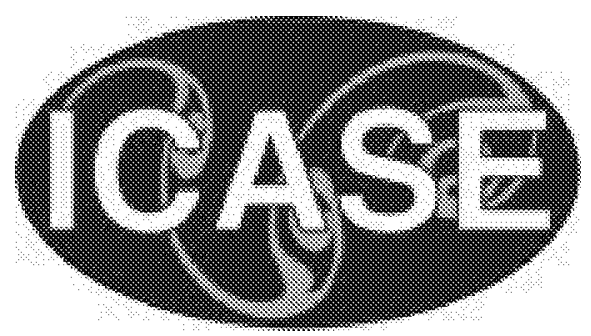

\title{
Theory of the Lattice Boltzmann Method: Dispersion, Dissipation, Isotropy, Galilean Invariance, and Stability
}

Pierre Lallemand

Université Paris-Sud, Orsay Cedex, France

Li-Shi Luo

ICASE, Hampton, Virginia 


\section{The NASA STI Program Office ... in Profile}

Since its founding, NASA has been dedicated to the advancement of aeronautics and space science. The NASA Scientific and Technical Information (STI) Program Office plays a key part in helping NASA maintain this important role.

The NASA STI Program Office is operated by Langley Research Center, the lead center for NASA's scientific and technical information. The NASA STI Program Office provides access to the NASA STI Database, the largest collection of aeronautical and space science STI in the world. The Program Office is also NASA's institutional mechanism for disseminating the results of its research and development activities. These results are published by NASA in the NASA STI Report Series, which includes the following report types:

- TECHNICAL PUBLICATION. Reports of completed research or a major significant phase of research that present the results of NASA programs and include extensive data or theoretical analysis. Includes compilations of significant scientific and technical data and information deemed to be of continuing reference value. NASA counter-part or peer-reviewed formal professional papers, but having less stringent limitations on manuscript length and extent of graphic presentations.

- TECHNICAL MEMORANDUM. Scientific and technical findings that are preliminary or of specialized interest, e.g., quick release reports, working papers, and bibliographies that contain minimal annotation. Does not contain extensive analysis.

- CONTRACTOR REPORT. Scientific and technical findings by NASA-sponsored contractors and grantees.
- CONFERENCE PUBLICATIONS. Collected papers from scientific and technical conferences, symposia, seminars, or other meetings sponsored or co-sponsored by NASA.

- SPECIAL PUBLICATION. Scientific, technical, or historical information from NASA programs, projects, and missions, often concerned with subjects having substantial public interest.

- TECHNICAL TRANSLATION. Englishlanguage translations of foreign scientific and technical material pertinent to NASA's mission.

Specialized services that help round out the STI Program Office's diverse offerings include creating custom thesauri, building customized databases, organizing and publishing research results ... even providing videos.

For more information about the NASA STI Program Office, you can:

- Access the NASA STI Program Home Page at http://www.sti.nasa.gov/STIhomepage.html

- Email your question via the Internet to help@sti.nasa.gov

- Fax your question to the NASA Access Help Desk at (301) 621-0134

- Phone the NASA Access Help Desk at (301) 621-0390

- Write to: NASA Access Help Desk NASA Center for AeroSpace Information 7121 Standard Drive Hanover, MD 21076-1320 
NASA/CR-2000-210103

ICASE Report No. 2000-17

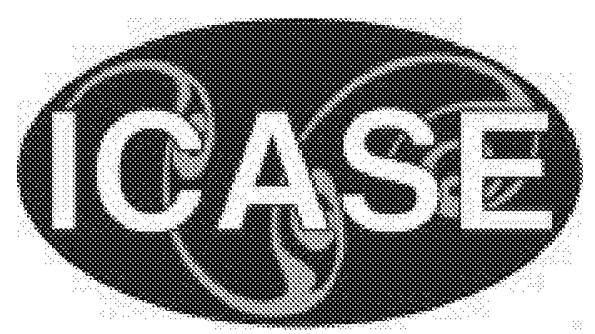

\section{Theory of the Lattice Boltzmann Method: Dispersion, Dissipation, Isotropy, Galilean Invariance, and Stability}

Pierre Lallemand

Université Paris-Sud, Orsay Cedex, France

Li-Shi Luo

ICASE, Hampton, Virginia

Institute for Computer Applications in Science and Engineering NASA Langley Research Center

Hampton, VA

Operated by Universities Space Research Association

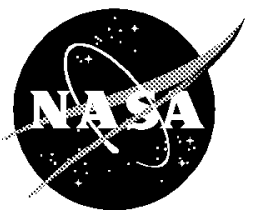

National Aeronautics and Space Administration

Langley Research Center

Hampton, Virginia 23681-2199
Prepared for Langley Research Center under Contract NAS 1-97046 
Available from the following:

NASA Center for AeroSpace Information (CASI)

7121 Standard Drive

Hanover, MD 21076-1320

(301) 621-0390
National Technical Information Service (NTIS)

5285 Port Royal Road

Springfield, VA 22161-2171

(703) 487-4650 


\title{
THEORY OF THE LATTICE BOLTZMANN METHOD: DISPERSION, DISSIPATION, ISOTROPY, GALILEAN INVARIANCE, AND STABILITY
}

\author{
PIERRE LALLEMAND* AND LI-SHI LUO ${ }^{\dagger}$
}

\begin{abstract}
The generalized hydrodynamics (the wave vector dependence of the transport coefficients) of a generalized lattice Boltzmann equation (LBE) is studied in detail. The generalized lattice Boltzmann equation is constructed in moment space rather than in discrete velocity space. The generalized hydrodynamics of the model is obtained by solving the dispersion equation of the linearized LBE either analytically by using perturbation technique or numerically. The proposed LBE model has a maximum number of adjustable parameters for the given set of discrete velocities. Generalized hydrodynamics characterizes dispersion, dissipation (hyper-viscosities), anisotropy, and lack of Galilean invariance of the model, and can be applied to select the values of the adjustable parameters which optimize the properties of the model. The proposed generalized hydrodynamic analysis also provides some insights into stability and proper initial conditions for LBE simulations. The stability properties of some 2D LBE models are analyzed and compared with each other in the parameter space of the mean streaming velocity and the viscous relaxation time. The procedure described in this work can be applied to analyze other LBE models. As examples, LBE models with various interpolation schemes are analyzed. Numerical results on shear flow with an initially discontinuous velocity profile (shock) with or without a constant streaming velocity are shown to demonstrate the dispersion effects in the LBE model; the results compare favorably with our theoretical analysis. We also show that whereas linear analysis of the LBE evolution operator is equivalent to Chapman-Enskog analysis in the long wave-length limit (wave vector $\boldsymbol{k}=\mathbf{0}$ ), it can also provide results for large values of $\boldsymbol{k}$. Such results are important for the stability and other hydrodynamic properties of the LBE method and cannot be obtained through Chapman-Enskog analysis.
\end{abstract}

Key words. kinetic method, lattice Boltzmann equation, derivation of hydrodynamic equation, stability analysis, numerical artifacts of the LBE method

\section{Subject classification. Physical Sciences}

1. Introduction. The method of lattice Boltzmann equation (LBE) is an innovative numerical method based on kinetic theory to simulate various hydrodynamic systems $[34,5,36]$. Although the LBE method was developed only a decade ago, it has attracted significant attention recently $[3,6]$, especially in the area of complex fluids including multi-phase fluids $[40,41,23,32,24,25]$, suspensions in fluid [35], and visco-elastic fluids $[12,13]$. The lattice Boltzmann equation was introduced to overcome some serious deficiencies of its historic predecessor: the lattice gas automata (LGA) $[10,46,11]$. The lattice Boltzmann equation circumvents two major shortcomings of the lattice gas automata: intrinsic noise and limited values of transport coefficients, both due to the Boolean nature of the LGA method. However, despite the notable success of the

${ }^{*}$ Laboratoire ASCI, Bâtiment 506, Université Paris-Sud (Paris XI Orsay), 91405 Orsay Cedex, France (email address: lalleman@asci.fr).

${ }^{\dagger}$ Institute for Computer Applications in Science and Engineering, Mail Stop 132C, NASA Langley Research Center, 3 West Reid Street, Building 1152, Hampton, VA 23681-2199 (email address: luo@icase.edu). This research was supported by the National Aeronautics and Space Administration under NASA Contract No. NAS1-97046 while the author was in residence at the Institute for Computer Applications in Science and Engineering (ICASE), NASA Langley Research Center, Hampton, VA 23681-2199. 
LBE method in simulating laminar $[27,31,16,17]$ and turbulent [45] flows, understanding of some important theoretical aspects of the LBE method, such as the stability of the LBE method, is still lacking. It was only very recently that the formal connections between the lattice Boltzmann equation and the continuous Boltzmann equation $[19,20,1]$ and other kinetic schemes [28] were established.

In this work we intend to study two important aspects of the LBE method which have not been systematically studied yet: (a) the dispersion effects due to the presence of a lattice space; (b) conditions for stability. We first construct a LBE model in moment space based upon the generalized lattice Boltzmann equation due to d'Humières [8]. The proposed model has a maximum number of adjustable parameters allowed by the freedom provided by a given discrete velocity set. These adjustable parameters are used to optimize the properties of the model through a systematic analysis of the generalized hydrodynamics of the model. Generalized hydrodynamics characterizes dispersion, dissipation (hyper-viscosities), anisotropy, lack of Galilean invariance, and instability of the LBE models in general. The proposed generalized hydrodynamic analysis enables us to improve the properties of the models in general. The analysis also provides us better insights into the conditions under which the LBE method is applicable and comparable to conventional CFD techniques.

Furthermore, from a theoretical perspective, we would like to argue that our approach can circumvent the Chapman-Enskog analysis to obtain the macroscopic equations from the LBE models $[8,12,13]$. The essence of our argument is that the validity of the Chapman-Enskog analysis is entirely based upon the fact that there are two disparate spatial scales in real fluids: the kinetic (mean-free-path) and the hydrodynamic scale the ratio of which is the Knudsen number. When the LBE method is used to simulate hydrodynamic motion over a few lattice spacings, there is no such separation of the two scales. Therefore, the applicability of Chapman-Enskog analysis to the LBE models might become dubious. Under the circumstances, analyzing the generalized hydrodynamics of the model becomes not only appropriate but also necessary.

It should also be pointed out that there exists previous work on the generalized hydrodynamics of the LGA models [33, 30, 15, 14, 7] and the LBE models [2]. However, the previous work only provides analysis on non-hydrodynamic behavior of the models at finite wave-length, without addressing important issues such as the instability of the LBE method or providing insights as how to construct better models. In the present work, by using a model with as many adjustable parameters as possible, we analyze the generalized hydrodynamics of the model so that we can identify the causes of certain non-hydrodynamic behavior, such as anisotropy, and lack of Galilean invariance, and instability. Therefore, the analysis shows how to improve the model in a systematic and coherent fashion.

This paper is organized as follows: Sec. 2 gives a brief introduction of the two-dimensional 9-velocity LBE model in discrete velocity space. Sec. 3 discusses the generalized LBE model in moment space. Sec. 4 derives the linearized lattice Boltzmann equation from the generalized LBE model. Sec. 5 analyzes the hydrodynamic modes of the linearized evolution operator of the generalized LBE model, and the generalized hydrodynamics of the model. The dispersion, dissipation, isotropy, and Galilean invariance of the model are discussed. The eigenvalue problem of the linearized evolution operator is solved analytically and numerically. Sec. 6 analyzes the stability of the LBE model with BGK approximation, and compares with the stability of the LBE model presented in this paper. Sec. 7 discusses the correct initial conditions in the LBE simulations, and presents numerical tests of shear flows with discontinuities in the initial velocity profile. Sec. 8 provides a summary and concludes the paper. Two appendices provide additional analysis for variations of the LBE models. Appendix A analyzes a model with coupling between density $\rho$ and velocity $\boldsymbol{u}$, and Appendix B analyzes the LBE models with various interpolation schemes. 
2. 2D 9-Velocity LBE Model. The guiding principle of the LBE models is to construct a dynamical system on a simple lattice of high symmetry (mostly square in 2D and cubic in 3D) involving a number of quantities which can be interpreted as the single particle distribution functions of fictitious particles on the links of the lattice. These quantities then evolve in a discrete time according to certain rules that are chosen to attain some desirable macroscopic behavior which emerges at scales large relative to the lattice spacing. One possible "desirable behavior" is that of a compressible thermal or athermal viscous fluid. (For simplicity of the analysis, we shall restrict our analysis to the athermal case in this work.) We shall demonstrate that the LBE models can satisfactorily mimic the fluid behavior to an extent that the models are indeed useful to simulate flows according to the similarity principle of fluid mechanics. For the sake of simplicity, we limit our discussions here in two-dimensional space. The extension to three-dimensional space is straightforward, albeit tedious.

A particular two-dimensional LBE model considered in this work is the 9-velocity model. In this model, space is discretized into square lattice, and there are nine discrete velocities given by:

$$
\boldsymbol{e}_{\alpha}= \begin{cases}(0,0), & \alpha=0, \\ (\cos [(\alpha-1) \pi / 2], \sin [(\alpha-1) \pi / 2]) c, & \alpha=1-4, \\ (\cos [(2 \alpha-9) \pi / 4], \sin [(2 \alpha-9) \pi / 4]) \sqrt{2} c, & \alpha=5-8,\end{cases}
$$

where $c=\delta_{x} / \delta_{t}$ is the unit of velocity, and $\delta_{x}$ and $\delta_{t}$ are the lattice constant of the lattice space and the unit of time (time step), respectively. From now on we shall use the units of $\delta_{x}=1$ and $\delta_{t}=1$ such that all the relevant quantities are dimensionless. The above discrete velocities correspond to the particle motion from a lattice node $\boldsymbol{r}_{j}$ to either itself, one of the 4 nearest neighbors $(\alpha=1-4)$, or one of the 4 next-nearest neighbors $(\alpha=5-8)$. This model can be easily extended to include more discrete velocities and in space of higher dimensions, thus to include further distant neighbors where the particles move to in one time step. Nevertheless, "hopping" to a neighbor on the lattice induces inherent limitations in the discretization of velocity space.

For the particular model discussed here, nine real numbers describe the medium at each node $\boldsymbol{r}_{j}$ of a square lattice:

$$
\left\{f_{\alpha}\left(\boldsymbol{r}_{j}\right) \mid \alpha=0,1, \ldots, 8\right\}
$$

The number $f_{\alpha}$ can be considered as the distribution function of velocity $\boldsymbol{e}_{\alpha}$ at location $\boldsymbol{r}_{j}$ (and at a particular time $t$ ). The set $\left\{f_{\alpha}\right\}$ can be represented by a vector in $\mathbb{R}^{9}$ which defines the state of the medium at each lattice node:

$$
\left|f\left(\boldsymbol{r}_{j}\right)\right\rangle \equiv\left(f_{0}, f_{1}, \ldots, f_{8}\right)^{\top}
$$

Once the vector $\left|f\left(\boldsymbol{r}_{j}\right)\right\rangle$ is given at a point $\boldsymbol{r}_{j}$ in space, the state of the medium at this point is fully specified.

The evolution of the medium occurs at discrete times $t=n \delta_{t}$, (with $\delta_{t}=1$ ). The evolution consists of two steps:

1. Motion to the relevant neighbors (modeling of advection);

2. Redistribution of the $\left\{f_{\alpha}\right\}$ at each nodes (modeling of collisions). These steps are described by the equation

$$
f_{\alpha}\left(r_{j}+e_{\alpha}, t+1\right)=f_{\alpha}\left(r_{j}, t\right)+\Omega_{\alpha}(f) .
$$


The above equation is the so-called Lattice Boltzmann equation (LBE). The lattice Boltzmann equation can be rewritten in a concise vector form:

$$
\left|f\left(\boldsymbol{r}_{j}+\boldsymbol{e}_{\alpha}, t+1\right)\right\rangle=\left|f\left(\boldsymbol{r}_{j}, t\right)\right\rangle+|\Delta f\rangle
$$

where the following notations are adopted:

$$
\begin{aligned}
& \left|f\left(\boldsymbol{r}_{j}+\boldsymbol{e}_{\alpha}, t+1\right)\right\rangle \equiv\left(f_{0}\left(\boldsymbol{r}_{j}+\boldsymbol{e}_{0}, t+1\right), f_{1}\left(\boldsymbol{r}_{j}+\boldsymbol{e}_{1}, t+1\right), \ldots, f_{8}\left(\boldsymbol{r}_{j}+\boldsymbol{e}_{8}, t+1\right)\right)^{\top}, \\
& |\Delta f\rangle \equiv\left(\Omega_{0}(f), \Omega_{1}(f), \ldots, \Omega_{8}(f)\right)^{\top},
\end{aligned}
$$

so that $\left|f\left(\boldsymbol{r}_{j}+\boldsymbol{e}_{\alpha}, t+1\right)\right\rangle$ is the vector of a state after advection, and $|\Delta f\rangle$ is the vector of the changes in $|f\rangle$ due to collision $\Omega$.

The advection is straightforward in the LBE models. The collisions represented by the operator $\Omega$ may be rather complicated. However, $\Omega$ must satisfy conservation laws and be compatible with the symmetry of the model (the underlying lattice space). This might simplify $\Omega$ considerably. One simple collision model is the BGK model $[4,5,36]$ :

$$
\Omega_{\alpha}=-\frac{1}{\tau}\left[f_{\alpha}-f_{\alpha}^{(\mathrm{eq})}\right]
$$

where $\tau$ is the relaxation time in unit of time step $\delta_{t}$ (which is set to be 1 here), and $f_{\alpha}^{\text {(eq) }}$ is the equilibrium distribution function which satisfies the following conservation conditions for an athermal medium:

$$
\begin{aligned}
& \rho=\sum_{\alpha} f_{\alpha}^{(\mathrm{eq})}=\sum_{\alpha} f_{\alpha}, \\
& \rho \boldsymbol{u}=\sum_{\alpha} \boldsymbol{e}_{\alpha} f_{\alpha}^{(\mathrm{eq})}=\sum_{\alpha} \boldsymbol{e}_{\alpha} f_{\alpha},
\end{aligned}
$$

where $\rho$ and $u$ are the (mass) density and the velocity of the medium at each lattice node, respectively. For the so-called 9-velocity BGK model, the equilibrium is usually taken as:

$$
f_{\alpha}^{(\text {eq })}=w_{\alpha} \rho\left[1+3\left(\boldsymbol{e}_{\alpha} \cdot \boldsymbol{u}\right)+\frac{9}{2}\left(\boldsymbol{e}_{\alpha} \cdot \boldsymbol{u}\right)^{2}-\frac{3}{2} \boldsymbol{u}^{2}\right],
$$

where $w_{0}=4 / 9, w_{1,2,3,4}=1 / 9$, and $w_{5,6,7,8}=1 / 36$.

Some shortcomings of the BGK model are apparent. For instance, because the model relies on a single relaxation parameter $\tau$, the Prandtl number must be unity when the model is applied to thermal fluids, among other things. One way to overcome these shortcomings of the BGK LBE model $[5,36]$ is to use a generalized LBE model which nevertheless retains the simplicity and computational efficiency of the BGK LBE model.

3. Moment Representation and Generalized 2D LBE. Given a set of $b$ discrete velocities, $\left\{\boldsymbol{e}_{\alpha} \mid \alpha=0,1, \ldots,(b-1)\right\}$ with corresponding distribution functions, $\left\{f_{\alpha} \mid \alpha=0,1, \ldots,(b-1)\right\}$, one can construct a $b$-dimensional vector space $\mathbb{R}^{b}$ based upon the discrete velocity set, and this is usually the space mostly used in the previous discussion of the LBE models. One can also construct a space based upon the (velocity) moments of $\left\{f_{\alpha}\right\}$. Obviously, there are $b$ independent moments for the discrete velocity set. The reason in favor of using the moment-representation is somewhat obvious. It is well understood in the context of kinetic theory that various physical processes in fluids, such viscous transport, can be approximantly described by coupling or interaction among 'modes' (of the collision operator), and these modes are directly related to the moments (e.g., the hydrodynamic modes are linear combinations of mass, and momenta 
moments). Thus the moment-representation provides a convenient and effective means to incorporate the physics into the LBE models. Because the physical significance of the moments is obvious (hydrodynamic quantities and their fluxes, etc.), the relaxation parameters of the moments are directly related to the various transport coefficients. This mechanism allows us to control each mode independently. This also overcomes some obvious deficiencies of the usual BGK LBE model, such as a fixed Prandtl number, which is due to a single relaxation parameter of the model.

For the 9-velocity LBE model, we choose following moments to represent the model:

$$
\begin{aligned}
|\rho\rangle & =(1,1,1,1,1,1,1,1,1)^{\top}, \\
|e\rangle & =(-4,-1,-1,-1,-1,2,2,2,2)^{\top}, \\
|\varepsilon\rangle & =(4,2,2,2,2,1,1,1,1)^{\top}, \\
\left|j_{x}\right\rangle & =(0,1,0,-1,0,1,-1,-1,1)^{\top}, \\
\left|q_{x}\right\rangle & =(0,-2,0,2,0,1,-1,-1,1)^{\top}, \\
\left|j_{y}\right\rangle & =(0,0,1,0,-1,1,1,-1,-1)^{\top}, \\
\left|q_{y}\right\rangle & =(0,0,-2,0,2,1,1,-1,-1)^{\top}, \\
\left|p_{x x}\right\rangle & =(0,1,-1,1,-1,0,0,0,0)^{\top}, \\
\left|p_{x y}\right\rangle & =(0,0,0,0,0,1,-1,1,-1)^{\top} .
\end{aligned}
$$

The above vectors are represented in the space $\mathbb{V}=\mathbb{R}^{9}$ spanned by the discrete velocities $\left\{\boldsymbol{e}_{\alpha}\right\}$, and they are mutually orthogonal to each other. These vectors are not normalized; this makes the algebraic expressions involving these vectors which follow simpler. Note that the above vectors have an explicit physical significance related to the moments of $\left\{f_{\alpha}\right\}$ in discrete velocity space: $|\rho\rangle$ is the density mode; $|e\rangle$ is the energy mode; $|\varepsilon\rangle$ is related to energy square; $\left|j_{x}\right\rangle$ and $\left|j_{y}\right\rangle$ correspond to the $x$ - and $y$-component of momentum (mass flux); $\left|q_{x}\right\rangle$ and $\left|q_{y}\right\rangle$ correspond to the $x$ - and $y$-component of energy flux; and $\left|p_{x x}\right\rangle$ and $\left|p_{x y}\right\rangle$ correspond to the diagonal and off-diagonal component of the stress tensor. The components of these vectors in discrete velocity space $\mathbb{V}=\mathbb{R}^{9}$ are constructed as follows:

$$
\begin{aligned}
|\rho\rangle_{\alpha} & =\left|e_{\alpha}\right|^{0}=1 \\
|e\rangle_{\alpha} & =-4\left|\boldsymbol{e}_{\alpha}\right|^{0}+3\left(e_{\alpha, x}^{2}+e_{\alpha, y}^{2}\right) \\
|\varepsilon\rangle_{\alpha} & =4\left|e_{\alpha}\right|^{0}-\frac{21}{2}\left(e_{\alpha, x}^{2}+e_{\alpha, y}^{2}\right)+\frac{9}{2}\left(e_{\alpha, x}^{2}+e_{\alpha, y}^{2}\right)^{2} \\
\left|j_{x}\right\rangle_{\alpha} & =e_{\alpha, x} \\
\left|q_{x}\right\rangle_{\alpha} & =\left[-5\left|\boldsymbol{e}_{\alpha}\right|^{0}+3\left(e_{\alpha, x}^{2}+e_{\alpha, y}^{2}\right)\right] e_{\alpha, x} \\
\left|j_{y}\right\rangle_{\alpha} & =e_{\alpha, y} \\
\left|q_{y}\right\rangle_{\alpha} & =\left[-5\left|\boldsymbol{e}_{\alpha}\right|^{0}+3\left(e_{\alpha, x}^{2}+e_{\alpha, y}^{2}\right)\right] e_{\alpha, y} \\
\left|p_{x x}\right\rangle_{\alpha} & =e_{\alpha, x}^{2}-e_{\alpha, y}^{2} \\
\left|p_{x y}\right\rangle_{\alpha} & =e_{\alpha, x} e_{\alpha, y} .
\end{aligned}
$$

Thus,

$$
\begin{aligned}
& \rho=\langle\rho \mid f\rangle=\langle f \mid \rho\rangle, \\
& e=\langle e \mid f\rangle=\langle f \mid e\rangle, \\
& \varepsilon=\langle\varepsilon \mid f\rangle=\langle f \mid \varepsilon\rangle,
\end{aligned}
$$




$$
\begin{aligned}
j_{x} & =\left\langle j_{x} \mid f\right\rangle=\left\langle f \mid j_{x}\right\rangle, \\
q_{x} & =\left\langle q_{x} \mid f\right\rangle=\left\langle f \mid q_{x}\right\rangle, \\
j_{y} & =\left\langle j_{y} \mid f\right\rangle=\left\langle f \mid j_{y}\right\rangle, \\
q_{y} & =\left\langle q_{y} \mid f\right\rangle=\left\langle f \mid q_{y}\right\rangle, \\
p_{x x} & =\left\langle p_{x x} \mid f\right\rangle=\left\langle f \mid p_{x x}\right\rangle, \\
p_{x y} & =\left\langle p_{x y} \mid f\right\rangle=\left\langle f \mid p_{x y}\right\rangle .
\end{aligned}
$$

Similar to $\left\{f_{\alpha}\right\}$, the set of the above moments can also be concisely represented by a vector:

$$
|\varrho\rangle \equiv\left(\rho, e, \varepsilon, j_{x}, q_{x}, j_{y}, q_{y}, p_{x x}, p_{x y}\right)^{\top} .
$$

There obviously exists a transformation matrix $M$ between $|\varrho\rangle$ and $|f\rangle$ such that:

$$
\begin{aligned}
& |\varrho\rangle=\mathrm{M}|f\rangle, \\
& |f\rangle=\mathrm{M}^{-1}|\varrho\rangle .
\end{aligned}
$$

In other words, the matrix $M$ transforms a vector in the vector space $\mathbb{V}$ spanned by the discrete velocities into a vector in the vector space $\mathbb{M}=\mathbb{R}^{b}$ spanned by the moments of $\left\{f_{\alpha}\right\}$. The transformation matrix $M$ is explicitly given by:

$$
\begin{aligned}
& \mathbf{M} \equiv\left(\begin{array}{l}
\langle\rho| \\
\langle e| \\
\langle\varepsilon| \\
\left\langle j_{x}\right| \\
\left\langle q_{x}\right| \\
\left\langle j_{y}\right| \\
\left\langle q_{y}\right| \\
\left\langle p_{x x}\right| \\
\left\langle p_{x y}\right|
\end{array}\right) \equiv\left(\begin{array}{rrrrrrrrr}
1 & 1 & 1 & 1 & 1 & 1 & 1 & 1 & 1 \\
-4 & -1 & -1 & -1 & -1 & 2 & 2 & 2 & 2 \\
4 & -2 & -2 & -2 & -2 & 1 & 1 & 1 & 1 \\
0 & 1 & 0 & -1 & 0 & 1 & -1 & -1 & 1 \\
0 & -2 & 0 & 2 & 0 & 1 & -1 & -1 & 1 \\
0 & 0 & 1 & 0 & -1 & 1 & 1 & -1 & -1 \\
0 & 0 & -2 & 0 & 2 & 1 & 1 & -1 & -1 \\
0 & 1 & -1 & 1 & -1 & 0 & 0 & 0 & 0 \\
0 & 0 & 0 & 0 & 0 & 1 & -1 & 1 & -1
\end{array}\right) \\
& \equiv\left(|\rho\rangle,|e\rangle,|\varepsilon\rangle,\left|j_{x}\right\rangle,\left|q_{x}\right\rangle,\left|j_{y}\right\rangle,\left|q_{y}\right\rangle,\left|p_{x x}\right\rangle,\left|p_{x y}\right\rangle\right)^{\top} .
\end{aligned}
$$

The rows of the transformation matrix $\mathrm{M}$ are organized in the order of the corresponding tensor, rather than in the order of the corresponding moment. The first three rows of $\mathrm{M}$ correspond to $\rho, e$, and $\varepsilon$, which are scalars or zeroth-order tensors, and they are zeroth, second, and fourth order moment of $\left\{f_{\alpha}\right\}$, respectively. The next four rows correspond to $j_{x}, q_{x}, j_{y}$, and $q_{y}$, which are vectors or first-order tensors, and $j_{x}$ and $j_{y}$ are the first order moments, whereas $q_{x}$ and $q_{y}$ are the third order ones. The last two rows represent the stress tensor, which are second order moments and second order tensors. Again, this can easily be generalized to models using a larger discrete velocity set, and thus higher order moments, and in three-dimensional space. The main difficulty when using the LBE method to simulate a real isotropic fluid is how to systematically eliminate as much as possible the effects due to the symmetry of the underlying lattice. We shall proceed to analyze some simple (but non-trivial) hydrodynamic situations, and to make the flows as independent of the lattice symmetry as possible.

Because the medium simulated by the model is athermal, the only conserved quantities in the system are density $\rho$ and linear momentum $j=\left(j_{x}, j_{y}\right)$. Collisions do not change the conserved quantities. Therefore, in the moment space $\mathbb{M}$, collisions have no effect on these three quantities. We should stress that the 
conservation of energy is not considered here because the model is constructed to simulate an athermal medium. Moreover we find that the 9-velocity model is inadequate to simulate a thermal medium because it cannot have an isotropic Fourier law for the diffusion of the heat. Although the conserved moments are not affected by collisions, the non-conserved moments are affected by collisions, which in turn cause changes in the gradients or fluxes of the conserved moments which are higher order moments. In what follows the modeling of the changes of the non-conserved moments is described.

Inspired by the kinetic theory for Maxwell molecules [26], we assume that the non-conserved moments relax linearly towards their equilibrium values that are functions of the conserved quantities. The relaxation equations for the non-conserved moments are prescribed as follows:

$$
\begin{aligned}
e^{*} & =e-s_{2}\left[e-e^{(\mathrm{eq})}\right], \\
\varepsilon^{*} & =\varepsilon-s_{3}\left[\varepsilon-\varepsilon^{(\mathrm{eq})}\right], \\
q_{x}^{*} & =q_{x}-s_{5}\left[q_{x}-q_{x}^{(\mathrm{eq})}\right], \\
q_{y}^{*} & =q_{y}-s_{7}\left[q_{y}-q_{y}^{(\mathrm{eq})}\right], \\
p_{x x}^{*} & =p_{x x}-s_{8}\left[p_{x x}-p_{x x}^{(\mathrm{eq})}\right], \\
p_{x y}^{*} & =p_{x y}-s_{9}\left[p_{x y}-p_{x y}^{(\mathrm{eq})}\right],
\end{aligned}
$$

where the quantities with and without superscript $*$ are post-collision and pre-collision values, respectively. The equilibrium values of the non-conserved moments in the above equations can be chosen at will provided that the symmetry of the problem is respected. We choose

$$
\begin{aligned}
e^{(\mathrm{eq})} & =\frac{1}{\langle e \mid e\rangle}\left[\alpha_{2}\langle\rho \mid \rho\rangle \rho+\gamma_{2}\left(\left\langle j_{x} \mid j_{x}\right\rangle j_{x}^{2}+\left\langle j_{y} \mid j_{y}\right\rangle j_{y}^{2}\right)\right] \\
& =\frac{1}{4} \alpha_{2} \rho+\frac{1}{6} \gamma_{2}\left(j_{x}^{2}+j_{y}^{2}\right), \\
\varepsilon^{(\mathrm{eq})} & =\frac{1}{\langle\varepsilon \mid \varepsilon\rangle}\left[\alpha_{3}\langle\rho \mid \rho\rangle \rho+\gamma_{4}\left(\left\langle j_{x} \mid j_{x}\right\rangle j_{x}^{2}+\left\langle j_{y} \mid j_{y}\right\rangle j_{y}^{2}\right)\right] \\
& =\frac{1}{4} \alpha_{3} \rho+\frac{1}{6} \gamma_{4}\left(j_{x}^{2}+j_{y}^{2}\right), \\
q_{x}^{(\mathrm{eq})} & =\frac{\left\langle j_{x} \mid j_{x}\right\rangle}{\left\langle q_{x} \mid q_{x}\right\rangle} c_{1} j_{x}=\frac{1}{2} c_{1} j_{x}, \\
q_{y}^{(\mathrm{eq})} & =\frac{\left\langle j_{y} \mid j_{y}\right\rangle}{\left\langle q_{y} \mid q_{y}\right\rangle} c_{1} j_{y}=\frac{1}{2} c_{1} j_{y}, \\
p_{x x}^{(\mathrm{eq})} & =\gamma_{1} \frac{1}{\left\langle p_{x x} \mid p_{x x}\right\rangle}\left(\left\langle j_{x} \mid j_{x}\right\rangle j_{x}^{2}-\left\langle j_{y} \mid j_{y}\right\rangle j_{y}^{2}\right)=\frac{1}{2} \gamma_{1}\left(j_{x}^{2}-j_{y}^{2}\right), \\
p_{x y}^{(\mathrm{eq})} & =\gamma_{3} \frac{\sqrt{\left\langle j_{x} \mid j_{x}\right\rangle\left\langle j_{y} \mid j_{y}\right\rangle}}{\left\langle p_{x x} \mid p_{x x}\right\rangle}\left(j_{x} j_{y}\right)=\frac{1}{2} \gamma_{3}\left(j_{x} j_{y}\right) .
\end{aligned}
$$

The values of the coefficients in the above equilibriums $\left(c_{1}, \alpha_{2,3}\right.$, and $\left.\gamma_{1,2,3,4}\right)$ will be determined in the next Section and summarized in Subsection 5.5. The choices of the above equilibriums are made based upon the inspection of the corresponding moments given by Eqs. (3.2), or the physical significance of these moments. Note that in principle $q_{x}$ and $q_{y}$ can include terms involving third order terms in terms of moment, such as $j_{x}^{3}$ and $j_{x} p_{x x}$ [13], and $\epsilon$ can include fourth order terms. Nevertheless, for the 9-velocity model, these terms of higher order are not considered because either they do not affect the hydrodynamics of the model significantly, or they lead to some highly anisotropic behavior which are undesirable for the LBE modeling of hydrodynamics. 
Clearly LBE modeling of fluids is rather different from real molecular dynamics. Therefore it is not necessary to try and solve the mathematically difficult problem to create an inter-particle collision mechanism for the fictitious particles in the LBE models that would give the same eigenmodes of the collision operator in the continuous Boltzmann equation. However, what can be accomplished is that by carefully crafting a simple model with certain degrees of freedom, we can optimize large scale properties of the model in the sense that generalized hydrodynamic effects (deviations from hydrodynamics) are minimized.

The values of the unknown parameters, $c_{1}, \alpha_{2,3}$, and $\gamma_{1,2,3,4}$, shall be determined by a study of the modes of the linearized collision operator with a periodic lattice of size $N_{x} \times N_{y}$.

It should be noted that in Eq. (3.8) the density $\rho$ does not appear in the terms quadratic in $j$. This implies that the density fluctuation is decoupled from the momentum equation, similar to an incompressible LBE model with a modified equilibrium distribution function [18]:

$$
f_{\alpha}^{(\text {eq })}=w_{\alpha}\left\{\rho+\rho_{0}\left[3\left(\boldsymbol{e}_{\alpha} \cdot \boldsymbol{u}\right)+\frac{9}{2}\left(\boldsymbol{e}_{\alpha} \cdot \boldsymbol{u}\right)^{2}-\frac{3}{2} \boldsymbol{u}^{2}\right]\right\}
$$

where the mean density $\rho_{0}$ is usually set to be 1 . The model corresponding to the equilibrium distribution function of Eq. (2.8) shall be analyzed in the Appendix A.

4. Linearized LBE. We consider the particular situation where the state of the medium is a flow specified by uniform and steady density $\rho$ (usually $\rho=1$ so the uniform density may not appear in subsequent expressions) and velocity in Cartesian coordinates $\boldsymbol{V}=\left(V_{x}, V_{y}\right)$, with a small fluctuation superimposed:

$$
|f\rangle=\left|f^{(0)}\right\rangle+|\delta f\rangle
$$

where $\left|f^{(0)}\right\rangle$ represents the uniform equilibrium state specified by uniform and steady density $\rho$ and velocity $\boldsymbol{V}=\left(V_{x}, V_{y}\right)$, and $|\delta f\rangle$ is the fluctuation. The linearized Boltzmann equation is:

$$
\left|\delta f\left(\boldsymbol{r}_{j}+\boldsymbol{e}_{\alpha}, t+1\right)\right\rangle=\left|\delta f\left(\boldsymbol{r}_{j}, t\right)\right\rangle+\Omega^{(0)}\left|\delta f\left(\boldsymbol{r}_{j}, t\right)\right\rangle
$$

where $\Omega^{(0)}$ is the linearized collision operator:

$$
\Omega_{\beta \alpha}^{(0)}=\left.\frac{\partial \Omega_{\alpha}}{\partial f_{\beta}}\right|_{|f\rangle=\left|f^{(0)}\right\rangle} \equiv \Omega_{\alpha, \beta}\left(\left\{f_{\alpha}^{(0)}\right\}\right) .
$$

In the moment space $\mathbb{M}$, the linearized collision operator can be easily obtained by using Eqs. (3.7) and (3.8):

$$
C_{\beta \alpha}=\left.\frac{\left\langle\varrho_{\beta} \mid \varrho_{\beta}\right\rangle}{\left\langle\varrho_{\alpha} \mid \varrho_{\alpha}\right\rangle} \frac{\partial \Delta \varrho_{\alpha}}{\partial \varrho_{\beta}}\right|_{|\varrho\rangle=\left|\varrho^{(0)}\right\rangle},
$$

where $\varrho_{\alpha}$ and $\left|\varrho_{\alpha}\right\rangle, \alpha=0,1, \ldots, b$, are the moments defined by Eqs. (3.3) and the corresponding vectors in $\mathbb{V}=\mathbb{R}^{9}$ defined by Eqs. (3.1); $\Delta \varrho_{\alpha}$ is the change of the moment due to collision given by Eqs. (3.7); $|\varrho\rangle=\left|\varrho^{(0)}\right\rangle$ is the vector of all moments at the uniform equilibrium state [see Eq. (3.4) for the definition of $|\varrho\rangle$. Obviously the linearized collision operator $C$ depends on the uniform state specified by density $\rho$ and velocity $\boldsymbol{V}=\left(V_{x}, V_{y}\right)$, upon which the perturbation $|\delta f\rangle$ is superimposed. Specifically, for the 9-velocity 
model,

$$
\mathrm{C}=\left(\begin{array}{ccccccccc}
0 & 0 & 0 & 0 & 0 & 0 & 0 & 0 & 0 \\
s_{2} \alpha_{2} / 4 & -s_{2} & 0 & s_{2} \gamma_{2} V_{x} / 3 & 0 & s_{2} \gamma_{2} V_{y} / 3 & 0 & 0 & 0 \\
s_{3} \alpha_{3} / 4 & 0 & -s_{3} & s_{3} \gamma_{4} V_{x} / 3 & 0 & s_{3} \gamma_{4} V_{y} / 3 & 0 & 0 & 0 \\
0 & 0 & 0 & 0 & 0 & 0 & 0 & 0 & 0 \\
0 & 0 & 0 & s_{5} c_{1} / 2 & -s_{5} & 0 & 0 & 0 & 0 \\
0 & 0 & 0 & 0 & 0 & 0 & 0 & 0 & 0 \\
0 & 0 & 0 & 0 & 0 & s_{7} c_{1} / 2 & -s_{7} & 0 & 0 \\
0 & 0 & 0 & 3 s_{8} \gamma_{1} V_{x} & 0 & -3 s_{8} \gamma_{1} V_{y} & 0 & -s_{8} & 0 \\
0 & 0 & 0 & 3 s_{9} \gamma_{3} V_{y} / 2 & 0 & 3 s_{9} \gamma_{3} V_{x} / 2 & 0 & 0 & -s_{9}
\end{array}\right) .
$$

The perturbation in the moments corresponding to $|\delta f\rangle$ is $|\delta \varrho\rangle$, and $|\delta \varrho\rangle=\mathrm{M}|\delta f\rangle$. The change of the perturbation due to collisions is linearly approximated by $|\Delta \varrho\rangle=\mathrm{C}|\delta \varrho\rangle$ in the moment space $\mathbb{M}$ spanned by $\left\{\left|\varrho_{\alpha}\right\rangle \mid \alpha=0,1, \ldots,(b-1)\right\}$. This change of state in discrete velocity space $\mathbb{V}$ is $|\Delta f\rangle=\mathrm{M}^{-1} \mathrm{C}|\delta \varrho\rangle$. Therefore the Eq. (4.2) becomes

$$
\left|\delta f\left(\boldsymbol{r}_{j}+\boldsymbol{e}_{\alpha}, t+1\right)\right\rangle=\left|\delta f\left(\boldsymbol{r}_{j}, t\right)\right\rangle+\mathrm{M}^{-1} \mathrm{CM}\left|\delta f\left(\boldsymbol{r}_{j}, t\right)\right\rangle .
$$

In Fourier space, the above equation becomes:

$$
\mathrm{A}|\delta f(\boldsymbol{k}, t+1)\rangle=\left[\mathrm{I}+\mathrm{M}^{-1} \mathrm{CM}\right]|\delta f(\boldsymbol{k}, t)\rangle,
$$

where $A$ is advection operator represented by the following diagonal matrix in discrete velocity space $\mathbb{V}=\mathbb{R}^{9}$ :

$$
\mathrm{A}_{\alpha \beta}=\exp \left(i \boldsymbol{e}_{\alpha} \cdot \boldsymbol{k}\right) \delta_{\alpha \beta},
$$

where $\delta_{\alpha \beta}$ is the Kronecker $\delta$. It should be noted that for a mode of wave number $\boldsymbol{k}=\left(k_{x}, k_{y}\right)$ in Cartesian coordinates, the advection operator $\mathrm{A}$ in the above equation can be written as follows:

$$
\mathrm{A}=\operatorname{diag}(1, p, q, 1 / p, 1 / q, p q, q / p, 1 / p q, p / q),
$$

where

$$
p=e^{i k_{x}}, \quad q=e^{i k_{y}} .
$$

The advection can be decomposed into two parts, along two orthogonal directions, such as $x$-axis and $y$-axis in Cartesian coordinates:

$$
\begin{aligned}
& \mathrm{A}\left(k_{x}\right) \equiv \mathrm{A}\left(k_{x}, k_{y}=0\right)=\operatorname{diag}(1, p, 1,1 / p, 1, p, 1 / p, 1 / p, p), \\
& \mathrm{A}\left(k_{y}\right) \equiv \mathrm{A}\left(k_{x}=0, k_{y}\right)=\operatorname{diag}(1,1, q, 1,1 / q, q, q, 1 / q, 1 / q) .
\end{aligned}
$$

and $\mathrm{A}\left(k_{x}\right)$ and $\mathrm{A}\left(k_{y}\right)$ commute with each other:

$$
\mathrm{A}=\mathrm{A}\left(k_{x}\right) \mathrm{A}\left(k_{y}\right)=\mathrm{A}\left(k_{y}\right) \mathrm{A}\left(k_{x}\right),
$$

i.e., the advection operation can be applied along $x$-direction first, and then along $y$-direction, or vice versa. The linearized evolution equation (4.7) can be further written in a concise form:

$$
|\delta f(\boldsymbol{k}, t+1)\rangle=\mathrm{L}|\delta f(\boldsymbol{k}, t)\rangle,
$$

where

$$
L \equiv A^{-1}\left[I+M^{-1} C M\right]
$$

is the linearized evolution operator. 


\section{Modes of Linearized LBE.}

5.1. Hydrodynamic modes and transport coefficients. The evolution equation (4.6) is a difference equation which has a general solution:

$$
\left|G\left(\boldsymbol{r}_{j}, t=l\right)\right\rangle=K_{x}^{m} K_{y}^{n} z^{l}\left|G_{0}\right\rangle
$$

where $m$ and $n$ are indices for space $\left(\boldsymbol{r}_{j}=m \hat{\boldsymbol{x}}+n \hat{\boldsymbol{y}}\right)$, and $\hat{\boldsymbol{x}}$ and $\hat{\boldsymbol{y}}$ are units vectors along the $x$-axis and $y$-axis, respectively; $\left|G_{0}\right\rangle$ is the initial state. We can consider the particular case of a periodic system such that the spatial dependence of the above general solution can be chosen as

$$
|\delta f\rangle=\exp \left(-i \boldsymbol{k} \cdot \boldsymbol{r}_{j}+z t\right)\left|G\left(\boldsymbol{r}_{j}, t\right)\right\rangle .
$$

By substituting Eqs. (5.1) and (5.2) into the linearized LBE (4.11), we obtain the following equation:

$$
z\left|G_{0}\right\rangle=\mathrm{L}\left|G_{0}\right\rangle
$$

The above equation leads to the dispersion relation between $z$ and $k$ :

$$
\operatorname{det}[\mathbf{L}-z \mathbf{I}]=0
$$

which determines the transport behaviors of various modes depending on the wave vector $\boldsymbol{k}$. The solution of the above eigenvalue problem of the linearized evolution operator $L$ provides not only the dispersion relation, but also the solution of the initial value problem of Eq. (4.11):

$$
|\delta f(\boldsymbol{k}, t)\rangle=\mathrm{L}^{t}|\delta f(\boldsymbol{k}, 0)\rangle=\sum_{\alpha=1}^{b} z_{\alpha}^{t}\left|z_{\alpha}\right\rangle\left\langle z_{\alpha} \mid \delta f(\boldsymbol{k}, 0)\right\rangle,
$$

where $\left|z_{\alpha}\right\rangle$ is the eigenvector of $\mathbf{L}$ with eigenvalues $z_{\alpha}$ in discrete velocity space $\mathbb{V}$.

The eigenvalue problem of Eq. (5.4) cannot be solved analytically in general, except for some very special cases. Nevertheless, it can be easily solved numerically using various packages for linear algebra, such as LAPACK. For small $\boldsymbol{k}$, it can be solved by a series expansion in $\boldsymbol{k}$. The only part in $\mathbf{L}$ which has $\boldsymbol{k}$-dependence is the advection operator $A$. Therefore, we can expand $A^{-1}$ in $L$ :

$$
\mathrm{K} \equiv \mathrm{A}^{-1}=\mathrm{K}^{(0)}+\mathrm{K}^{(1)}(\boldsymbol{k})+\mathrm{K}^{(2)}\left(\boldsymbol{k}^{2}\right)+\cdots+\mathrm{K}^{(n)}\left(\boldsymbol{k}^{n}\right)+\cdots,
$$

where $\mathrm{K}^{(n)}$ depends on $\boldsymbol{k}^{n}$ :

$$
\mathrm{K}_{\alpha \beta}^{(n)}=\frac{1}{n !}\left(-i \boldsymbol{k} \cdot \boldsymbol{e}_{\alpha}\right)^{n} \delta_{\alpha \beta} .
$$

When $\boldsymbol{k}=\mathbf{0}$, the eigenvalue problem of the $(b \times b)$-matrix $\mathrm{L}^{(0)}=\left(\mathbf{I}+\mathbf{M}^{-1} \mathrm{CM}\right)$ can be solved analytically. There exists an eigenvalue of 1 with three-fold degeneracy, which corresponds to three hydrodynamic (conserved) modes in the system: one transverse (shear) and two longitudinal (sound) modes. It is interesting to note that when $\boldsymbol{k}=(\pi, 0)$ or $\boldsymbol{k}=(0, \pi)$, L also has an eigenvalue of -1 , which corresponds to the checkerboard mode, i.e., it is a conserved mode of $\mathrm{L}^{2}$. Being a neutral mode as far as stability is concerned, it will be necessary to study how it is affected by a mean velocity $\boldsymbol{V}$. Thus we shall have to analyze the model for $k$ ranging from 0 to $\pi$, which the standard Chapman-Enskog analysis cannot do.

The hydrodynamic modes at $\boldsymbol{k}=\mathbf{0}$ are:

$$
\begin{aligned}
& \left|\varrho_{T}\right\rangle=\cos \theta\left|j_{x}\right\rangle-\sin \theta\left|j_{y}\right\rangle \equiv\left|j_{T}\right\rangle, \\
& \left|\varrho_{ \pm}\right\rangle=|\rho\rangle \pm\left(\cos \theta\left|j_{x}\right\rangle+\sin \theta\left|j_{y}\right\rangle\right) \equiv|\rho\rangle \pm\left|j_{L}\right\rangle,
\end{aligned}
$$


where $\theta$ is the polar angle of wave vector $\boldsymbol{k}$. For finite $\boldsymbol{k}$, the behavior of these hydrodynamic modes depends upon $\boldsymbol{k}$. In two-dimensional space, these linearized hydrodynamic modes behave as follows [29]:

$$
\begin{aligned}
& \left|\varrho_{T}(t)\right\rangle=z_{T}^{t}\left|\varrho_{T}(0)\right\rangle=\exp [-i k(g V \cos \phi) t] \exp \left(-\nu k^{2} t\right)\left|\varrho_{T}(0)\right\rangle, \\
& \left|\varrho_{ \pm}(t)\right\rangle=z_{ \pm}^{t}\left|\varrho_{ \pm}(0)\right\rangle=\exp \left[ \pm i k\left(c_{s} \pm g V \cos \phi\right) t\right] \exp \left[-(\nu / 2+\eta) k^{2} t\right]\left|\varrho_{ \pm}(0)\right\rangle,
\end{aligned}
$$

where $\nu$ and $\eta$ are the shear and bulk viscosity, respectively; the coefficient $g$ indicates whether system is Galilean invariant (that $g=1$ implies Galilean invariance); $c_{s}$ is the sound speed; $V$ is the magnitude of the uniform streaming velocity of the system $\boldsymbol{V}=\left(V_{x}, V_{y}\right)$; and $\phi$ is angle between the streaming velocity $\boldsymbol{V}$ and the wave vector $\boldsymbol{k}$. The Galilean-coefficient $g(\boldsymbol{k})$ is similar to the $g$-factor in the FHP lattice gas automata $[10,46,11]$, which also determines the Galilean invariance of the system. The transport coefficients and the Galilean-coefficient are related to the eigenvalues of $L$ as the following:

$$
\begin{aligned}
& \nu(\boldsymbol{k})=-\frac{1}{k^{2}} \operatorname{Re}\left[\ln z_{T}(\boldsymbol{k})\right], \\
& g(\boldsymbol{k}) V \cos \phi=-\frac{1}{k} \operatorname{Im}\left[\ln z_{T}(\boldsymbol{k})\right], \\
& \frac{1}{2} \nu(\boldsymbol{k})+\eta(\boldsymbol{k})=-\frac{1}{k^{2}} \operatorname{Re}\left[\ln z_{ \pm}(\boldsymbol{k})\right], \\
& c_{s}(\boldsymbol{k}) \pm g(\boldsymbol{k}) V \cos \phi=\mp \frac{1}{k} \operatorname{Im}\left[\ln z_{ \pm}(\boldsymbol{k})\right],
\end{aligned}
$$

where $z_{T}(\boldsymbol{k})$ and $z_{ \pm}(\boldsymbol{k})$ are the eigenvalues corresponding to the hydrodynamic modes of the linearized evolution operator $\mathrm{L}$. Since the transport coefficients can be obtained through a perturbation analysis, we shall use the following series expansion in $\boldsymbol{k}$ :

$$
\begin{aligned}
\nu(\boldsymbol{k}) & =\nu_{0}-\nu_{1} k^{2}+\ldots+(-1)^{n} \nu_{n} k^{2 n}+\ldots, \\
\eta(\boldsymbol{k}) & =\eta_{0}-\eta_{1} k^{2}+\ldots+(-1)^{n} \eta_{n} k^{2 n}+\ldots, \\
c_{s}(\boldsymbol{k}) & =C_{0}-C_{1} k^{2}+\ldots+(-1)^{n} C_{n} k^{2 n}+\ldots, \\
g(\boldsymbol{k}) & =g_{0}-g_{1} k^{2}+\ldots+(-1)^{n} g_{n} k^{2 n}+\ldots
\end{aligned}
$$

It should be noted that, in the usual Chapman-Enskog analysis of LBE models, one only obtains the values of the transport coefficients at $\boldsymbol{k}=\mathbf{0}$. As we shall demonstrate later, higher order corrections to the transport coefficients (i.e., hyper-viscosities) are important to the LBE hydrodynamics, especially for spatial scales of a few lattice spacings.

One possible method to solve the dispersion relation $\operatorname{det}[\mathbf{L}-z \mathbf{l}]=0$ is to apply the Gaussian elimination technique using $1 / s_{i}$ as small parameters for the non-conserved modes (the kinetic modes). Starting from a $9 \times 9(b \times b$ in general $)$ determinant, we obtain a $3 \times 3$ determinant for the 3 conserved modes. The elements of this new determinant are computed as series of $1 / s_{i}$ and $k$ with the necessary numbers of terms to achieve a given accuracy when computing the roots of the dispersion equation.

It should be mentioned that the interest of the present technique is that it provides a very simple means to analyze models with various streaming and collision rules with as many adjustable parameters as possible to be determined later when trying to satisfy either the stability criteria or physical requirements to model various hydrodynamic systems. Free parameters are the equilibrium coefficients in Eqs. (3.8): $c_{1}, \alpha_{i}$, and $\gamma_{i} ;$ and relaxation rates $s_{i}$.

5.2. Case with no streaming velocity $(V=0)$. We first consider the case in which the streaming velocity $\boldsymbol{V}=\mathbf{0}$. To the first order in $\boldsymbol{k}$, we obtain two solutions of $\operatorname{Im}\left(\ln z_{ \pm}\right)=\mp i k c_{s}$ with

$$
c_{s}^{2}=\frac{1}{3}\left(2+\frac{\alpha_{2}}{8}\right) \text {. }
$$


These are the sound modes supported by the medium. At the next order, we obtain modes with $\operatorname{Re}\left(\ln z_{T}\right)=$ $-\nu_{0} k^{2}$. To enforce isotropy we need to have

$$
\frac{1}{s_{9}}-\frac{1}{2}=2\left(\frac{1}{s_{8}}-\frac{1}{2}\right) \frac{\left(c_{1}+4\right)}{\left(2-c_{1}\right)},
$$

such that the $\theta$-dependence in $\nu_{0}$ vanishes,

$$
\nu_{0}=\frac{\left(2-c_{1}\right)}{12}\left(\frac{1}{s_{8}}-\frac{1}{2}\right),
$$

which can be interpreted as the shear viscosity of the medium in the limit $\boldsymbol{k}=\mathbf{0}$ (measured in basic units of space and time). For the sound modes, we also find an attenuation rate $\operatorname{Re}\left(\ln z_{ \pm}\right)=-\left(\nu_{0} / 2+\eta_{0}\right) k^{2}$ where $\left(\nu_{0} / 2+\eta_{0}\right)$ is the longitudinal kinematic viscosity in a two-dimensional system. The bulk viscosity of the model at long wave length limit $\boldsymbol{k}=\mathbf{0}$ is:

$$
\eta_{0}=\frac{\left(c_{1}+10-12 c_{s}^{2}\right)}{24}\left(\frac{1}{s_{2}}-\frac{1}{2}\right)
$$

The positivity of the transport coefficients leads to the bounds on the adjustable parameters:

$$
\begin{aligned}
-16 & <\alpha_{2}, \\
-4 & <c_{1}<2,
\end{aligned}
$$

and the bounds on the following relaxation parameters:

$$
\begin{aligned}
& 0<s_{2}<2 \\
& 0<s_{8}<2
\end{aligned}
$$

The bounds for $\alpha_{2}$ and $c_{1}$ will be further narrowed in the following analysis. Based upon the above results of $\nu_{0}, \eta_{0}$, and $c_{s}$, it is clear that the model is isotropic at rest (i.e., the streaming velocity $\boldsymbol{V}=\mathbf{0}$ ) and in the limit of $\boldsymbol{k}=\mathbf{0}$. The Galilean-coefficient $g$ cannot be determined when the streaming velocity $\boldsymbol{V}=\mathbf{0}$. Therefore, the case of a finite streaming velocity $\boldsymbol{V}$ is considered next.

5.3. Case with a constant streaming velocity $\boldsymbol{V}$. As indicated by Eqs. (5.9), to the first order in $\boldsymbol{k}$, the three hydrodynamic roots of the dispersion equation $\left(z_{T}\right.$ and $\left.z_{ \pm}\right)$give the phase $g V \cos \phi$ and the sound speed $c_{s}$. In order to make the root of the transverse mode $\left(z_{T}\right)$ to have a correct phase corresponding to the streaming velocity $\boldsymbol{V}$, as expected for a model satisfying Galilean invariance, i.e., $g_{0}=1$, we must set

$$
\gamma_{1}=\gamma_{3}=\frac{2}{3}
$$

If we further set

$$
\gamma_{2}=18
$$

then we obtain the roots of the sound modes $\left(z_{ \pm}\right)$which lead to the sound speed

$$
C_{s}=V \cos \phi \pm \sqrt{c_{s}^{2}+V^{2} \cos ^{2} \phi},
$$

where $V \cos \phi \equiv \boldsymbol{V} \cdot \hat{\boldsymbol{k}}$, and $\hat{\boldsymbol{k}}$ is the unit vector parallel to $\boldsymbol{k}$. This clearly shows that the system obeys Galilean invariance only up to first order in $\boldsymbol{V}$. One way to correct this defect is to allow for compressibility effects in the equilibrium properties, as shown in Appendix A. The dispersion of sound can be computed 
either analytically, by carrying out the perturbation expansion in $\boldsymbol{k}$ or numerically, by solving the eigenvalue problem for any value of $\boldsymbol{k}$. The dispersion of sound is important when studying the nonlinear acoustic properties of the medium.

Second, the attenuation of transverse wave depends not only on $\boldsymbol{V}$ but also on the direction of the wave vector $\boldsymbol{k}$. In order to eliminate the anisotropy in the $\boldsymbol{V}$-dependence of the shear wave attenuation, we must choose:

$$
c_{1}=-2 .
$$

With the above choice of $c_{1}$, the shear viscosity in the limit of $\boldsymbol{k}=\mathbf{0}$ is given by:

$$
\begin{aligned}
\nu_{0}= & {\left[s_{2}\left(2-s_{8}\right)\left(c_{s}^{2}+\left(1-3 c_{s}^{2}\right) V^{2} \cos ^{2} \phi\right)+3\left(2\left(s_{8}-s_{2}\right)\right.\right.} \\
& \left.\left.+s_{8}\left(s_{2}-2\right) \cos ^{2} \phi\right) V^{4} \cos ^{2} \phi\right] /\left[6 s_{2} s_{8}\left(V^{2} \cos ^{2} \phi+c_{s}^{2}\right)\right] .
\end{aligned}
$$

Similarly, from the attenuation of acoustic waves, one obtains the bulk viscosity (in the limit of $\boldsymbol{k}=\mathbf{0}$ ) which has a complicated dependence on the streaming velocity $\boldsymbol{V}$ :

$$
\begin{aligned}
\eta_{0}= & \left\{V \operatorname { c o s } \phi \sqrt { V ^ { 2 } \operatorname { c o s } ^ { 2 } \phi + c _ { s } ^ { 2 } } \left[12 V^{2}\left(\left(s_{2}-s_{8}\right)+s_{2}\left(s_{8}-2\right) \cos ^{2} \phi\right)\right.\right. \\
& \left.+\left(2 s_{2}-3 s_{2} s_{8}+4 s_{8}\right)\left(1-3 c_{s}^{2}\right)\right] \\
& +3 V^{4} \cos ^{2} \phi\left[\cos ^{2} \phi\left(2 s_{8}+3 s_{2} s_{8}-8 s_{2}\right)+6\left(s_{2}-s_{8}\right)\right] \\
& +2 V^{2} \cos ^{2} \phi\left[6\left(s_{2} s_{8}-s_{2}-s_{8}\right) c_{s}^{2}+s_{8}\left(2-s_{2}\right)\right] \\
& \left.+c_{s}^{2}\left[6 V^{2}\left(s_{2}-s_{8}\right)+s_{8}\left(2-s_{2}\right)\left(2-3 c_{s}^{2}\right)\right]\right\} /\left\{12 s_{2} s_{8}\left(V^{2} \cos ^{2} \phi+c_{s}^{2}\right)\right\} .
\end{aligned}
$$

It is obvious that the streaming velocity $\boldsymbol{V}$ has a second order effect on $\nu_{0}$, and a first order effect on $\eta_{0}$. A careful inspection of the above result of $\eta_{0}$ indicates that the first order effect of $\boldsymbol{V}$ on $\eta_{0}$ can be eliminated by setting $c_{s}^{2}=1 / 3$ (or equivalently $\alpha_{2}=-8$ ). Furthermore, the second order effect of $\boldsymbol{V}$ on the sound speed and the longitudinal attenuation can also be eliminated by using a slightly more complicated model with thirteen velocities, as noted by a previous work [37].

In summary, although all the transport coefficients are isotropic in the limit $\boldsymbol{k}=\mathbf{0}$, some undesirable features of the LBE models can be clearly observed at the second order in $\boldsymbol{k}$ when the streaming velocity $\boldsymbol{V}$ has a finite magnitude. First, the acoustic wave propagation is not Galilean invariant. Second, both the shear and the bulk viscosity depend on $\boldsymbol{V}$. Nevertheless, these effects are of the second order in $\boldsymbol{V}$, and can be improved to higher order in both $\boldsymbol{k}$ and $\boldsymbol{V}$ by incorporating compressibility into the equilibrium properties of the moments (see Appendix A) or using models with a larger velocity set.

5.4. Third order result. The analysis in the previous subsections shows that isotropy for the hydrodynamic modes of the dispersion equation can be attained to the first and second order in $\boldsymbol{k}$ by carefully adjusting the parameters in the model. In the situation with a uniform streaming velocity $\boldsymbol{V}$ parallel to $\boldsymbol{k}$, we find that the third order term in $\boldsymbol{k}$ for the shear mode is anisotropic, i.e.,

$$
\begin{aligned}
g_{1}= & -\left(\frac{2}{3 s_{8}^{2}}-\frac{2}{3 s_{8}}+\frac{1}{9}\right)+\left(\frac{1}{3}-\frac{2}{s_{8}}+\frac{2}{s_{8}^{2}}\right) V^{2} \cos ^{2} \phi \\
& +\left[\frac{1}{3}-\frac{1}{s_{8}}+\frac{1}{s_{5}}\left(\frac{2}{s_{8}}-1\right)\right]\left(\cos ^{4} \theta-\cos ^{2} \theta+\frac{1}{3}\right) .
\end{aligned}
$$

The anisotropic term in $g_{1}$ (depending on $\cos \theta$ ) can be eliminated if we choose

$$
s_{5}=3 \frac{\left(2-s_{8}\right)}{\left(3-s_{8}\right)}
$$


As indicated by Eq. (5.13), parameter $s_{8}$ is usually chosen close to 2 from below in order to obtain a small shear viscosity (consequently large Reynolds number). Therefore, the preceding expression yields a small value for $s_{5}$. This would lead to an undesirable consequence: Mode $\left|q_{x}\right\rangle$ relaxed with the relaxation parameter $s_{5}$ would become a quasi-conserved mode leading to some sort of visco-elastic effect [13]. Therefore we usually choose to have large $s_{5}$ such that the advection coefficient of transverse waves has an angular dependence for non-zero $\boldsymbol{k}$ in third order in $\boldsymbol{k}$. That is, the physical conservation laws are preserved at the expense of the isotropy of the dispersion in third order (and all higher order) in $\boldsymbol{k}$.

It should be noted that the value of $g$ has effects on the Reynolds number because the time $t$ needs to be rescaled as $g t$.

5.5. Optimization of the model and connection to the BGK LBE model. Among seven adjustable parameters $\left(c_{1}, \alpha_{i}\right.$, and $\left.\gamma_{i}\right)$ in the equilibrium values of the moments in the model [see Eqs. (3.8)], so far only five of these parameters have been fixed by enforcing the model to satisfy certain basic physics as shown in the previous analysis: $c_{1}=-2, \alpha_{2}=-8, \gamma_{1}=\gamma_{3}=2 / 3$, and $\gamma_{2}=18$. These parameter values are the optimal choice in the sense that they yield the desirable properties (isotropy, Galilean invariance, etc.) to the highest order possible in wave vector $\boldsymbol{k}$. It should be stressed that the constraints imposed by isotropy and Galilean invariance are beyond the conservation constraints - models with only conservation constraints would not necessarily be isotropic and Galilean invariant in general, as observed in some newly proposed LBE model for non-ideal gases [44, 43, 32]. Two other parameters, $\alpha_{3}$ and $\gamma_{4}$, remain adjustable. In addition, there are six relaxation parameters $s_{i}$ in the model as opposed to one in the LBE BGK model. Two of them, $s_{2}$ and $s_{8}$ determine the bulk and the shear viscosity, respectively. Also, because $c_{1}=-2$, therefore $s_{9}=s_{8}$ [see Eq. (5.12)]. The remaining three relaxation parameters, $s_{3}, s_{5}$, and $s_{7}$ can be adjusted without having any effects on the transport coefficients in the order of $\boldsymbol{k}^{2}$. However, they do have effects in higher order terms. Therefore, one can keep values of these three relaxation parameters only slightly larger than 1 (no severe over-relaxation effects are produced by these modes) such that the corresponding kinetic modes are well separated from those modes more directly affecting hydrodynamic transport.

It is interesting to note that the present model degenerates to the BGK LBE model $[5,36]$ if we use a single relaxation parameter for all the modes, i.e., $s_{i}=1 / \tau$, and choose

$$
\begin{aligned}
\alpha_{3} & =4, \\
\gamma_{4} & =-18
\end{aligned}
$$

Therefore, in the BGK LBE model, all the modes relax with exactly the same relaxation parameter so there is no separation in time scales among the kinetic modes. This may severely affect the dynamics and the stability of the system, due to the coupling among these modes.

6. Local Stability Analysis. The stability of the LBE method has not been well understood, although there exists some preliminary work $[42,47]$. However, previous work does not provide much theoretical insight into either the causes or the remedies for the instability of the LBE method. In the following analysis, a systematic procedure which identifies some causes of instability is discussed and illustrated by some examples.

Our stability analysis relies on the eigenvalue problem for the linearized evolution operator $\mathbf{L}$, the dispersion equation. For large values of $\boldsymbol{k}$, one could in principle analyze the dispersion equation to higher order by perturbation expansion. In practice, it is more efficient to compute the roots of the dispersion equation numerically. We shall try to identify the conditions under which one of the modes becomes unstable: instability occurs when $\operatorname{Re}\left(\ln z_{\alpha}\right)<0$. 
TABLE 6.1

Special properties of the dispersion relation when the wave vector $\boldsymbol{k}$ is of some special values.

\begin{tabular}{|c|l|c|}
\hline$k$ & dispersion equation & conditions \\
\hline \multirow{5}{*}{$(0,0)$} & {$[z-1]^{3}=0$} & \\
& {$\left[z-\left(1-s_{2}\right)\right]=0$} & $s_{7}=s_{5}$ \\
& {$\left[z-\left(1-s_{3}\right)\right]=0$} & \\
& {$\left[z-\left(1-s_{5}\right)\right]^{2}=0$} & \\
& {$[z+1]=0$} & \\
& {$\left[z+\left(1-s_{5}\right)\right]=0$ or $\left[z+\left(1-s_{7}\right)\right]=0$} & \\
$( \pm 1,0) \pi$ & {$\left[z+\left(1-s_{8}\right)\right]=0$ or $\left[z+\left(1-s_{9}\right)\right]=0$} & \\
or & {$\left[z^{2}-\frac{1}{3} s_{5} z+s_{5}-1\right]=0$} & \\
$(0, \pm 1) \pi$ & {$\left[z^{4}+\frac{1}{3}\left(s_{3}-2 s_{2}\right) z^{3}\right.$} & \\
& $+\frac{1}{9}\left\{s_{2}\left(s_{8}-4 s_{3}\right)-6 s_{3} s_{8}+9\left(s_{2}+s_{3}+s_{8}-2\right)\right\} z^{2}$ & \\
& $+\frac{1}{3}\left(s_{8}-1\right)\left(s_{2}\left(s_{3}-2\right)+s_{3}\right) z$ & \\
& $\left.+\left(1-s_{2}\right)\left(1-s_{3}\right)\left(1-s_{8}\right)\right]=0$ & \\
& {$\left[z-\left(1-s_{8}\right)\right]^{2}=0$} & \\
& {$\left[z^{2}-\frac{1}{3} s_{5} z+s_{5}-1\right]^{2}=0$} & \\
$( \pm 1, \pm 1) \pi$ & {$\left[z^{3}+\frac{1}{9}\left(11 s_{2}-3 s_{3}-9\right) z^{2}\right.$} & \\
& $+\frac{1}{9}\left\{3\left(4 s_{3}-3\right)-s_{2}\left(s_{3}+2\right)\right\}$ & \\
& $\left.+\left(1-s_{2}\right)\left(1-s_{3}\right)\right]=0$ & \\
\hline
\end{tabular}

We have noticed some interesting qualitative properties of the dispersion for the 9-velocity model when wave vector $\boldsymbol{k}$ is parallel to certain special directions with respect to the lattice line. These properties are listed in Table 6 . These qualitative behaviors of the dispersion equation already demonstrate the strong anisotropy of the dispersion relations dictated by the lattice symmetry.

To exhibit the complex behavior of the dispersion equation, we compute the roots of the dispersion equation with a given set of parameters. Figures $1(\mathrm{a})$ and $1(\mathrm{~b})$ show the real and imaginary parts of the logarithm of the eigenvalues as functions of $\boldsymbol{k}$, respectively. Figs. 1 clearly exhibit the coalescence and branching of the roots. This suggests a complicated interplay between the modes of collision operator affecting the stability of the model. The asymmetric feature of these curves is due to the presence of a constant streaming.

The growth rate of a mode $\left|z_{\alpha}\right\rangle, \operatorname{Re}\left(\ln z_{\alpha}\right)$, depends on all the adjustable parameters: the relaxation parameters, the streaming velocity $\boldsymbol{V}$, and the wave vector $\boldsymbol{k}$. To illustrate this dependence, we consider the BGK LBE model with $1 / \tau=1.99$. Figure 2 shows the growth rate for the most unstable mode as a function of streaming velocity $\boldsymbol{V}$ and wave vector $\boldsymbol{k}$. For each $\boldsymbol{V}$, we let $\boldsymbol{k}$ be parallel to $\boldsymbol{V}$, with a polar angle $\theta$ with respect to the $x$-axis. Then we search for the most unstable mode in the interval $0 \leq k \leq \pi$. For the 9 -velocity BGK LBE model, the unstable mode starts to appear above $V \approx 0.07$. Figure 2 shows the strong anisotropy of the unstable mode: the growth rate significantly depends on the direction of $\boldsymbol{k}$, and the critical value of $\boldsymbol{k}$ at which the unstable mode starts to appear is also strongly anisotropic. We also compute the growth rate for the most unstable mode with $\boldsymbol{V}$ perpendicular to $\boldsymbol{k}$, and find that the stability of the model is generally qualitatively the same as when $\boldsymbol{V}$ is parallel to $\boldsymbol{k}$, but is slightly more stable. Generally we find that the transverse mode is more stable than longitudinal modes. In many instances we have observed 

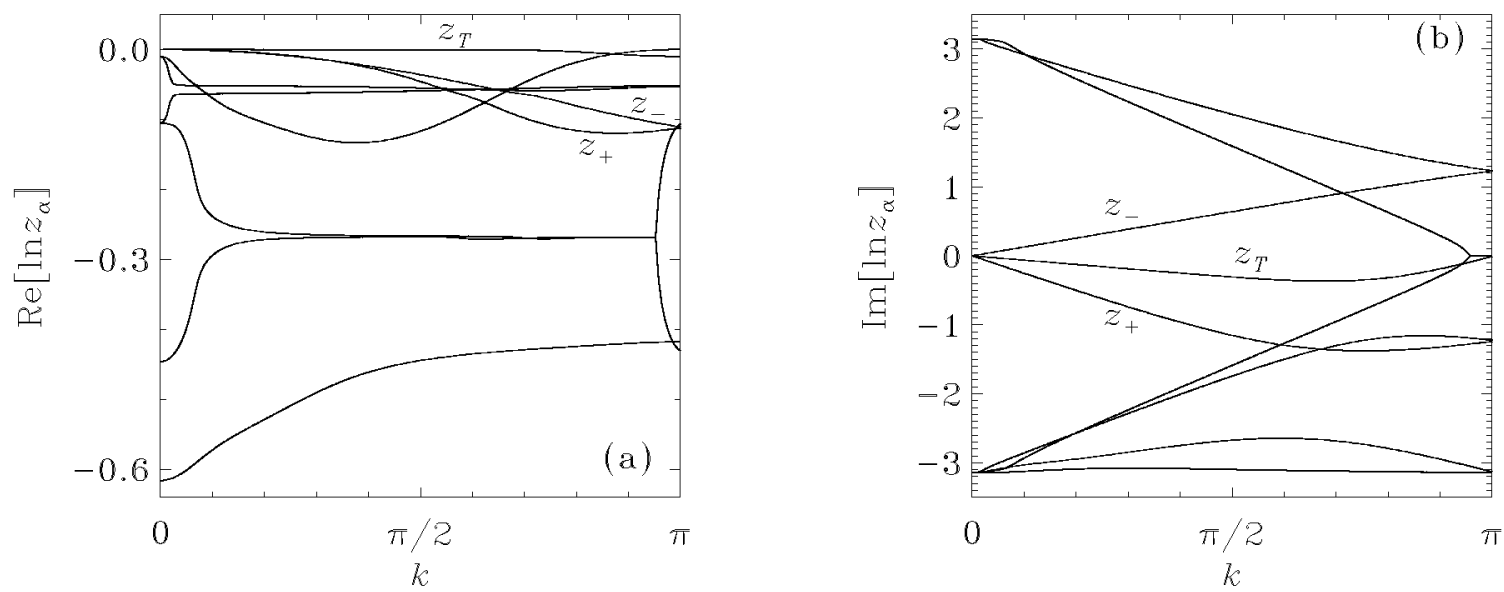

FIG. 1. The logarithmic eigenvalues of the 9-velocity model. The values of the parameters are: $\alpha_{2}=-8, \alpha_{3}=4$, $c_{1}=-2, \gamma_{1}=\gamma_{3}=2 / 3, \gamma_{2}=18$, and $\gamma_{4}=-18$. The relaxation parameters are: $s_{2}=1.64, s_{3}=1.54, s_{5}=s_{7}=1.9$, and $s_{8}=s_{9}=1.99$. The streaming velocity $\boldsymbol{V}$ is parallel to $\boldsymbol{k}$ with $V=0.2$, and $\boldsymbol{k}$ is along $x$-axis. $(a) \operatorname{Re}\left(\ln z_{\alpha}\right)$, and $(b) \operatorname{Im}\left(\ln z_{\alpha}\right)$.

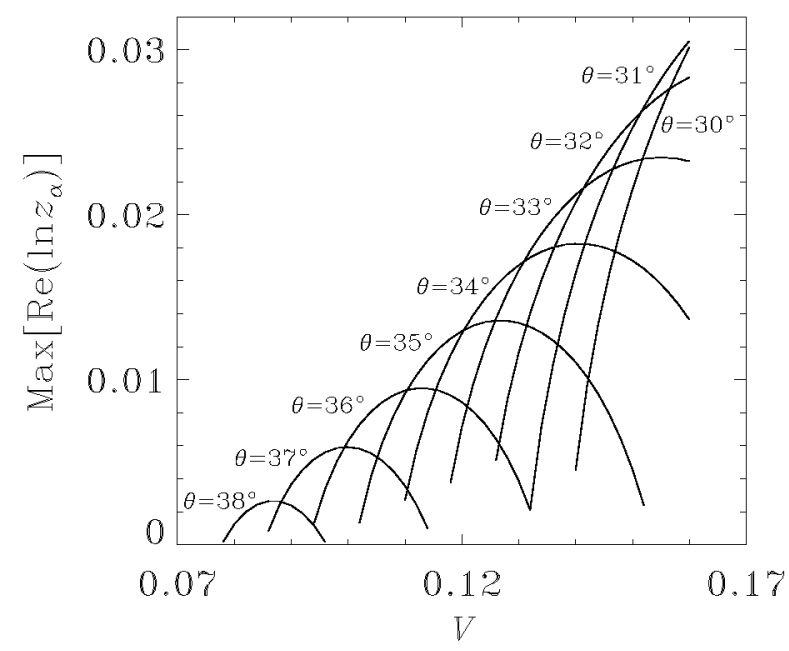

FIG. 2. The growth rate of the most unstable mode for the BGK LBE model $-\ln z_{\alpha}$ vs. the streaming velocity magnitude $V$. The relaxation parameter $s_{8}=1 / \tau=1.99$. The wave vector $\boldsymbol{k}$ is set parallel to the streaming velocity $\boldsymbol{V}$. For each value of $\boldsymbol{V}$ with a polar angle $\theta$ with respect to $x$-axis, the growth rate is computed in the interval $0<k \leq \pi$ in $k$-space. Each curve corresponds to the growth rate of the most unstable mode with a given $\boldsymbol{V}$, and $\boldsymbol{k}$ parallel to $\boldsymbol{V}$ with the polar angle $\theta$ with respect to $x$-axis.

that sound waves propagating in the direction of the mean flow velocity $\boldsymbol{V}$ can be quite unstable. This instability may be reduced by making the first order $\boldsymbol{V}$-dependent term in the attenuation of the sound waves $\left[\eta_{0}\right.$ in Eq. (5.22)] equal to 0 by choosing $c_{s}^{2}=1 / 3$, as indicated in the previous section. It should be noted that when the growth rate is infinitesimal, it takes extremely long time for the instability to develop in simulations. Because the unstable modes we have observed have a large wave vector $k$ (small spatial scale), therefore, as a practical means to reduce the effect of instabilities in LBE simulations, some kind of spatial or temporal filtering technique may be used in the LBE schemes to reduce small scale fluctuations and thus to limit the development of instabilities.

It should be pointed out that we do not discuss here the influence of boundary conditions which may completely change the stability behavior of the model through either large scale genuine hydrodynamic 


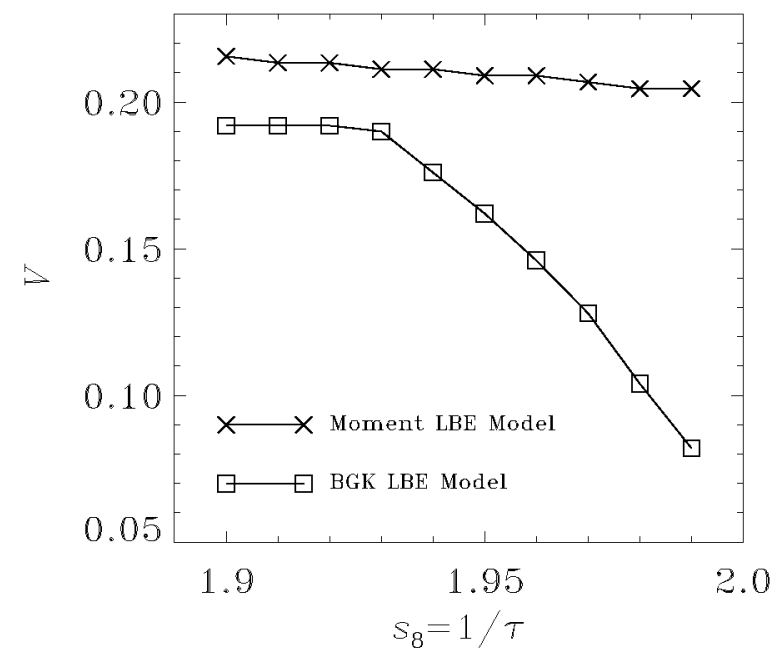

FIG. 3. The stability of the generalized $L B E$ model vs. the BGK LBE model in the parameter space of $V$ and $s_{8}=1 / \tau$. The line with symbol $\square$ and $\times$ are results for the BGK LBE model and the model proposed in this work, respectively. The region under a curve is the stable region in the parameter space of $V$ and $s_{8}=1 / \tau$. Note that the stability of the $B G K L B E$ model starts to deteriorate after $s_{8} \geq 1.92$, whereas the stability of the proposed generalized LBE model remains virtually intact.

behavior or local excitation of Knudsen modes.

As previously indicated, the adjustable parameters in our model can be used to alter the properties of the model. The stability of the BGK LBE model and our model is compared in Fig. 3. In this case we choose the adjustable parameters in our model to be the same as the BGK LBE model, but maintain the freedom of different modes to relax with different relaxation parameters $s_{i}$. Fig. 2 shows that for each given value of $\boldsymbol{V}$, there exists a maximum value of $s_{8}=1 / \tau$ (which determines the shear viscosity) below which there is no unstable mode. The values of other relaxation parameters used in our model are: $s_{2}=1.63$, $s_{3}=1.14, s_{5}=s_{7}=1.92$, and $s_{9}=s_{8}=1 / \tau$. Fig. 3 clearly shows that our model is more stable than the BGK LBE model in the interval $1.9 \leq s_{8}=1 / \tau \leq 1.99$. Therefore, we can conclude that by carefully separating the kinetic modes with different relaxation rates, we can indeed improve the stability of the LBE model significantly.

7. Numerical Simulations of Shear Flow Decay. To illustrate the dispersion effects on the shear viscosity in hydrodynamic simulations using the LBE method, we conduct a series of numerical simulations of the shear flow decay with different initial velocity profiles. The numerical implementation of the model is discussed next.

7.1. Numerical implementation and initial conditions. The evolution of the model still consists in two steps: advection and collision. The advection is executed in discrete velocity space, namely to $|f(\boldsymbol{x}, t)\rangle$, but not to the moments $|\varrho(\boldsymbol{x}, t)\rangle$. However, the collision is executed in moment space. Therefore, the evolution involves transformation between discrete velocity space $\mathbb{V}$ and moment space $\mathbb{M}$, similar to Fourier transform in the spectral or Galerkin methods. The evolution equation of the model is:

$$
\left|f\left(\boldsymbol{x}+\boldsymbol{e}_{\alpha} \delta_{t}, t+\delta_{t}\right)\right\rangle=|f(\boldsymbol{x}, t)\rangle+\mathrm{M}^{-1} \mathrm{~S}\left[|\varrho(\boldsymbol{x}, t)\rangle-\left|\varrho^{(\mathrm{eq})}\right\rangle\right],
$$

where $\mathbf{S}$ is the diagonal relaxation matrix:

$$
\mathrm{S} \equiv \operatorname{diag}\left(0,-s_{2},-s_{3}, 0,-s_{5}, 0,-s_{7},-s_{8},-s_{9}\right) .
$$


In simulations using the LBE method, the initial conditions provided are usually specified by velocity and pressure (density) fields. Often the initial condition of $f_{\alpha}$ is set to its equilibrium value corresponding to the given flow fields, with a constant density if the initial pressure field is not specified. The initial conditions of $f_{\alpha}$ can include the first order effect $f_{\alpha}^{(1)}$. The first order effect in moment space is obtained through Eq. (7.1):

$$
\left|\varrho^{(1)}\right\rangle=\mathrm{S}^{-1} \mathrm{MD}\left|f^{(\mathrm{eq})}\right\rangle
$$

where $\mathrm{D}$ is a diagonal differential operator:

$$
\mathrm{D}_{\alpha \beta}=\delta_{\alpha \beta} e_{\alpha} \cdot \nabla
$$

Eq. (7.3) is similar to Chapman-Enskog analysis of $f_{\alpha}^{(1)}$.

For the shear flow, only the initial velocity profile is given. The density mode is set to be uniform initially. The remaining modes are initialized as the following:

$$
\begin{aligned}
\rho & =1, \\
e & =-2+3\left(u_{x}^{2}+u_{y}^{2}\right), \\
\varepsilon & =1-3\left(u_{x}^{2}+u_{y}^{2}\right), \\
q_{x} & =-u_{x} \\
q_{y} & =-u_{y} \\
p_{x x} & =\left(u_{x}^{2}-u_{y}^{2}\right)-\frac{2}{3 s_{8}}\left(\partial_{x} u_{x}-\partial_{y} u_{y}\right), \\
p_{x y} & =u_{x} u_{y}-\frac{1}{3 s_{8}}\left(\partial_{y} u_{x}+\partial_{x} u_{y}\right) .
\end{aligned}
$$

The terms in $p_{x x}$ and $p_{x y}$ involving derivatives of the velocity field take into account of viscous effect in the initial conditions. These terms are obtained through Eq. (7.3). The first order terms in turn induce second order contributions (with respect to space derivatives) which are not included here. This leads to weak transients of short duration if there is separation of time scales $\left(2-s_{8}\right) \ll\left(2-s_{5}\right)$.

Our first test is the decay of a sinusoidal wave in a periodic system for various values of $\boldsymbol{k}$. The numerical and theoretical results agree with each other extremely well and confirm the $\boldsymbol{k}$-dependence of $g$ and $\nu$. The agreement indicates that our local analysis is indeed sufficiently accurate in this case.

The next case considered is more interesting and revealing because the initial velocity contains shocks. Consider a periodic domain of size $N_{x} \times N_{y}=84 \times 4$. At time $t=0$, we take a shear wave $u_{y}(x, 0)$ of rectangular shape (discontinuities in $u_{y}$ at $x=N_{x} / 4$ and $x=3 N_{x} / 4$ ):

$$
\begin{aligned}
& u_{y}(x, 0)=U_{0}, \quad 1<x \leq N_{x} / 4, \quad 3 N_{x} / 4<x \leq N_{x}, \\
& u_{y}(x, 0)=-U_{0}, \quad N_{x} / 4<x \leq 3 N_{x} / 4 .
\end{aligned}
$$

The initial condition $u_{x}(x, 0)$ is set to zero every where. We consider two separate cases with and without a constant streaming velocity $\boldsymbol{V}$.

7.2. Steady case $(\boldsymbol{V}=\mathbf{0})$. For the case of zero streaming velocity, the initial condition for $u_{x}$ is zero in the system. The solution of the Navier-Stokes equation for this simple problem is:

$$
u_{y}(x, t)=\sum_{n} a_{n} \exp \left(-\nu_{n} k_{n}^{2} t\right) \cos \left(k_{n} x\right),
$$



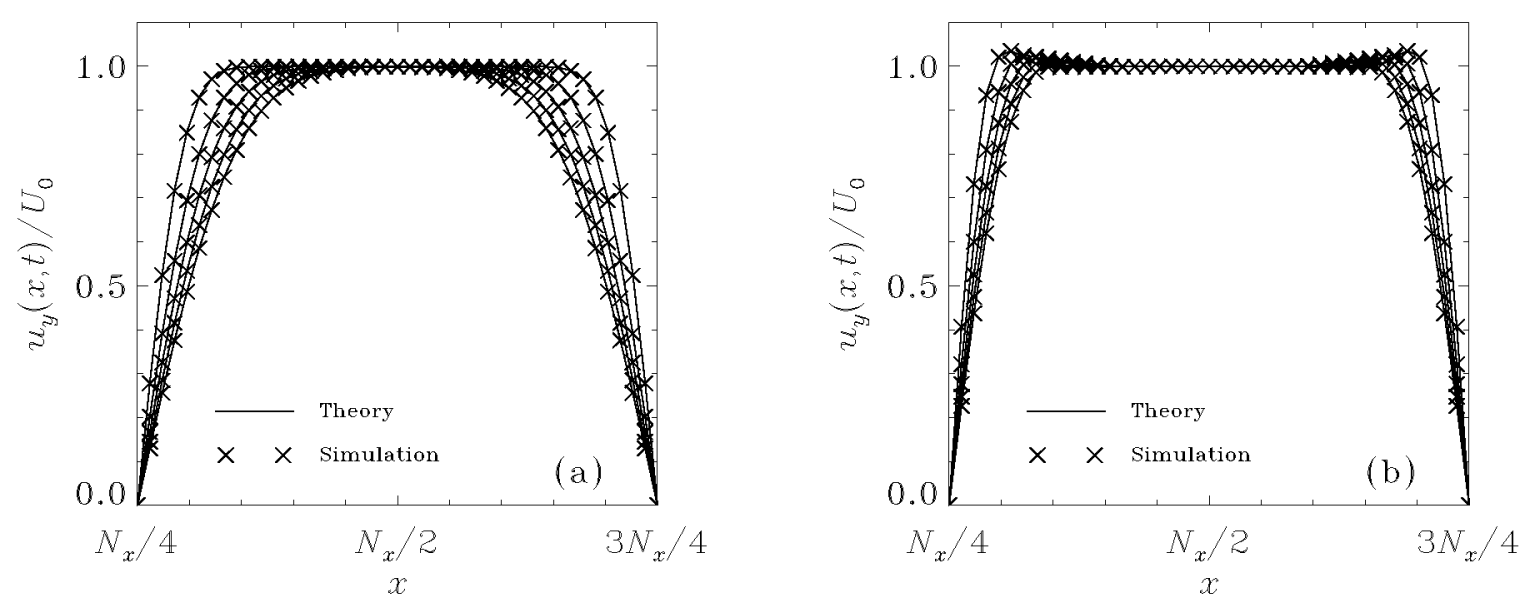

FIG. 4. Decay of discontinuous shear wave velocity profile $u_{y}(x, t)$. The lines and symbols $(\times)$ are theoretical [Eq. (7.6)] and numerical results, respectively. Only the positive half of the velocity profiles are shown in the Figures. (a) The LBE model with no interpolation, (b) with the central interpolation and $r=0.5$.

where $a_{n}$ are the Fourier coefficients of the initial velocity profile $u_{y}(x, 0), \nu_{n} \equiv \nu\left(k_{n}\right)$, and $k_{n}=2 \pi(2 n-$ $1) / N_{x}$. The magnitude of the $u_{y}(x, 0), U_{0}=0.0001$ in the simulations.

Figures 4(a) and 4(b) show the decay of the rectangular shear wave simulated by the normal LBE scheme and the LBE scheme with second-order central interpolation (with $r=0.5$, where $r$ is the ratio between advection length $\delta_{x}$ and grid size $\Delta_{x}$ ), respectively. (The detailed analysis of LBE schemes with various interpolations is provided in Appendix B.) The lines are theoretical results of Eq. (7.6) with $\nu\left(k_{n}\right)$ obtained numerically. The times at which the profile of $u_{y}(x, t)$ (normalized by $U_{0}$ ) shown in Figs. 4 are $t=100,200$, $\ldots, 500$. The numerical and theoretical results agree closely with each other. The close agreement shows the accuracy of the theory. In Fig. 4(b), the overshoots at early times due to the discontinuous initial condition are well captured by the analysis. This overshoot is entirely due to the strong $\boldsymbol{k}$-dependence of $\nu(\boldsymbol{k})$ caused by the interpolation. This phenomena is not necessarily connected to the Burnett effect, as claimed by a previous work [38]. This artifact is also commonly observed in other CFD methods involving interpolations.

Figure 5 shows the decay of $u_{y}(x, t)$ at one location of discontinuity, $x=3 N_{x} / 4=63$. We tested the normal LBE scheme without interpolation and the LBE scheme with second order central interpolation with $r=0.5$, and compared the numerical results with theoretical ones. Again, the numerical and theoretical results very well agree with each other for both cases (with and without interpolation). Note that the time is rescaled as $r^{-2} t$ in the Figure. It should be pointed out that the LBE solutions of the flow differ from the analytic solution of the Navier-Stokes equation in both short time and long time behavior. Interpolation causes overshoot in the velocity at the initial stage. Even without interpolation, the LBE solution does not decay (exponentially) right away. This is due to the variation of the viscosity with $\boldsymbol{k}$ and this could be interpreted as the influence of the kinetic modes. (If we had a vanishingly small Knudsen number then the $\boldsymbol{k}$-dependence would be negligible, however all relaxation rates must be smaller than 2 so that higher modes can play a role). This transient behavior is due to the higher order effect (of velocity gradient), as discussed previously.

7.3. Streaming case ( $V=$ constant $)$. We also consider the case with a constant streaming in the initial velocity, i.e., $u_{x}(x, 0)=V_{x}=0.08$. This allows us to check the effects of the non-Galilean invariance 


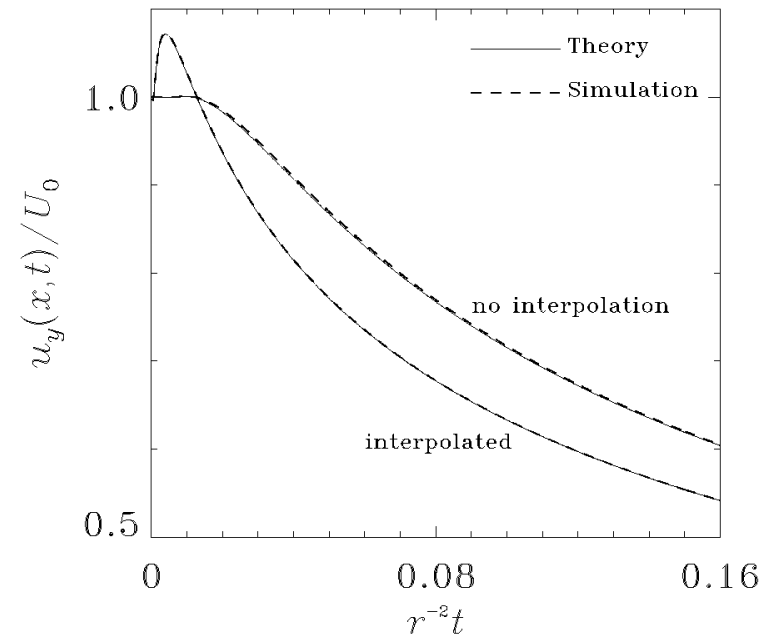

FIG. 5. Decay of discontinuous shear wave velocity $u_{y}(x, t)$ at a location close to the discontinuity $x=3 N_{x} / 4$. The solid lines and symbols $(\times)$ are theoretical and numerical results, respectively. The LBE scheme with no interpolation does not have a overshooting, whereas the LBE scheme with the central interpolation and $r=0.5$ has. The time is rescaled as $r^{-2} t$.

in the system. With a constant streaming velocity, the solution of the Navier-Stokes equation is:

$$
u_{y}(x, t)=\sum_{n} a_{n} \exp \left(-\nu_{n} k_{n}^{2} t\right) \cos \left[k_{n}\left(x-g_{n} V_{x} t\right)\right]
$$

where $g_{n} \equiv g\left(k_{n}\right)$ is the Galilean-coefficient.

Similar to Figs. 4, Figs. 6 show the evolution of $u_{y}(x, t)$ for the same times as in Figs. 4 . The solid lines and the symbols $(X)$ represent theoretical and numerical results, respectively. Shocks move from left to right with a constant velocity $V_{x}=0.08$. Figs. $6(\mathrm{a}), 6(\mathrm{~b})$, and $6(\mathrm{c})$ show the results for the normal LBE scheme without interpolation, the scheme with second order central interpolation, and the scheme with second order upwind interpolation, respectively. In Figs. 6(b) and 6(c), the dotted-lines are the results obtained by setting $g_{n}=1$ in Eq. (7.7). Clearly, the effect of $g(\boldsymbol{k})$ is significant. For the LBE scheme with central interpolation, the results in Fig. 6(b) with $g(\boldsymbol{k})=1$ under-predict the overshooting at the leading edge of the shock and over-predict the overshooting at the trailing edge; whereas the results in Fig. 6(c) for the LBE scheme with upwind interpolation over-predict the overshooting at the leading edge of the shock and under-predict the overshooting at the trailing edge.

8. Conclusion and Discussion. In this paper, a generalized 9-velocity LBE model based on the generalized LBE model of d'Humières [8] is presented. The model has the maximum number of adjustable parameters allowed by the discrete velocity set. The value of the adjustable parameters are obtained by optimizing the hydrodynamic properties of the model through the linear analysis of the LBE evolution operator. The linear analysis also provides the generalized hydrodynamics of the LBE model, from which dispersion, dissipation, isotropy, and stability of the model can be easily analyzed. In summary, a systematic and general procedure to analyze the LBE models is described in detail in this paper. Although the model studied in this paper is relatively simple, the proposed procedure can be readily applied to analyze more complicated LBE models.

The theoretical analysis of the model is verified through numerical simulation of various flows. The theoretical results closely predict the numerical results. The stability of the model is also analyzed and compared with the BGK LBE model. It is found that the mechanism of separate relaxations for the kinetic 

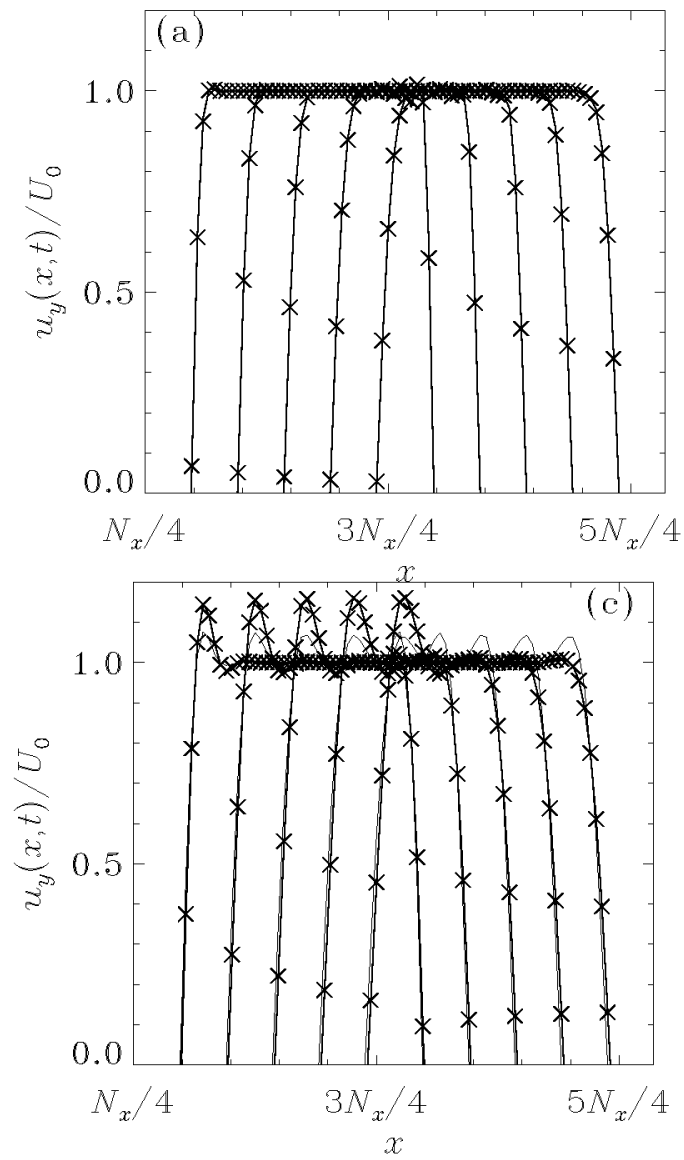

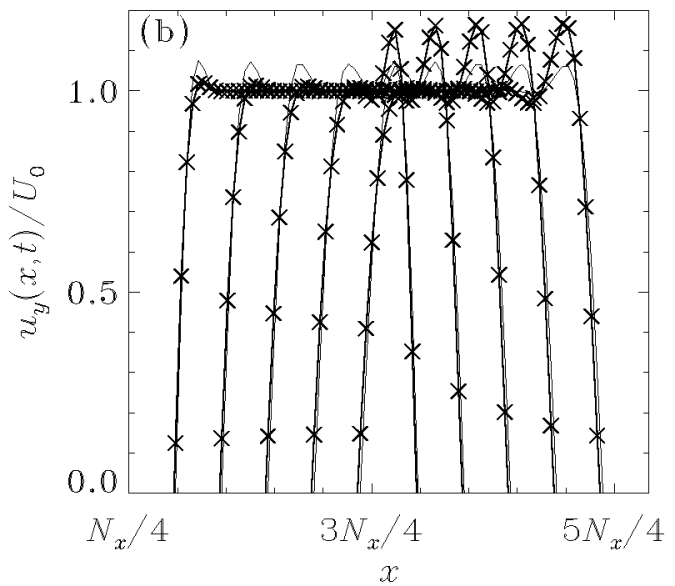

FIG. 6. Decay of discontinuous shear wave velocity profile $u_{y}(x, t)$ with a constant streaming velocity $V_{x}=0.08$. The thick lines and symbols $(\times)$ are theoretical [Eq. (7.7)] and numerical results, respectively. The thin lines in (b) and (c) are obtained by setting $g_{n}=1$ in Eq. (7.7). (a) The LBE model with no interpolation, (b) with the central interpolation and $r=0.5$, (c) with the upwind interpolation and $r=0.5$.

modes leads to a model which is much more stable than the BGK LBE model.

The proposed model is a Galerkin type of scheme. In comparison with the BGK LBE model, the proposed model requires the transformations between the discrete velocity space $\mathbb{V}$ and the moment space $\mathbb{M}$ back and forth in each step in the evolution equation. However, the extra computational cost due to this transformation is only about $10-20 \%$ of the total computing time. Thus, the computational efficiency is comparable to the BGK LBE model. Our analysis also shows that the LBE models with interpolation schemes have enormous numerical hyper-viscosities and anisotropies due to the interpolations.

We also find optimal features of the proposed 9-velocity model: it is difficult to improve the model by simply adding more velocities. For instance, we found that adding eight more velocities $( \pm 1, \pm 2)$ and $( \pm 2, \pm 1)$ would not improve the isotropy of the model. However, our analysis does not provide any a priori knowledge of an optimal set of discrete velocities. That problem can only be solved by optimization of the moment problem in velocity space [20]. It is also worth noting that the values of the adjustable parameters in our model coincide with the corresponding parameters in the BGK LBE model except two $\left(\alpha_{3}\right.$ and $\left.\gamma_{4}\right)$. The main distinction between our model and the BGK LBE model is that in our model has the freedom to allow the kinetic modes to relax differently, whereas in the BGK LBE model, all kinetic modes relax at the same rate. This mechanism severely affects the stability of the BGK LBE schemes especially when the 
system is strongly over-relaxed.

It should be mentioned that the procedure we propose here can be applied to analyze the linear stability of spatially nonuniform flows, such as Couette flow, Poiseuille flow, or lid-driven cavity flow. For spatially nonuniform flows, the lattice Boltzmann equation is linearized over a finite domain including boundary conditions. This leads to an eigenvalue problem with many more degrees of freedom as was needed in the analysis of this paper. Standard Arnoldi techniques [39] allow us to determine parts of the spectrum of the linearized collision operator, in particular to study the flow stability. This analysis enables us to understand the observation that some flows are much more stable than what is predicted by the linear analysis of spatially uniform flows. For instance, in plane Couette flow with only 2 nodes along the flow direction, the only possible values of $k$ along the same direction are 0 and $\pi$, which are far away from the value of $k$ at which the bulk instability occurs. Namely, the reciprocal lattice $\boldsymbol{k}$ is not large enough to accommodate the possible unstable modes. Furthermore, in the direction perpendicular to the flow, although the reciprocal lattice $\boldsymbol{k}$ can accommodate unstable shear modes, the velocity gradient, however, alters the stability of the system. (It improves the stability in this particular case.)

One philosophic point must be stressed. We deliberately did not derive the macroscopic equations corresponding to the LBE model in this work; instead, we only analyzed the generalized hydrodynamic behavior of the modes of the linearized LBE evolution operator. We argue that if the hydrodynamic modes behave exactly the same way as those of the linearized Navier-Stokes equations, up to a certain order of $\boldsymbol{k}$, provided that the Galilean invariance is also assured up to a certain order of $\boldsymbol{k}$, then we can claim that the LBE model is indeed adequate to simulate the Navier-Stokes equations (up to a certain order of $\boldsymbol{k}$ ). There is no distinction between the LBE model and the Navier-Stokes equations up to a certain order of $\boldsymbol{k}$. Thus, there is no need to use the Chapman-Enskog analysis to obtain the macroscopic equations from the LBE models. On the other hand, we have also shown that, in the limit of $\boldsymbol{k}=\mathbf{0}$, these two approaches obtain the same results in terms of the transport coefficients and the Galilean-coefficient. Nevertheless, it is very difficult to apply the Chapman-Enskog analysis to obtain the generalized hydrodynamics of the LBE models, which is important to LBE numerical simulations of hydrodynamic systems. The stability result obtained by the linear analysis presented in this paper is very difficult for the standard Chapman-Enskog analysis to obtain. Therefore, the proposed procedure to analyze the LBE model indeed contains more information and is more general than the low order Chapman-Enskog analysis. Albeit its generality and powerfulness, the linear analysis has its limitations. Because it is a local analysis, it does not deal with gradients.

Our future work is to extend the analysis to fully thermal and compressible LBE models in threedimensional space.

Acknowledgments. PL would like to acknowledge the support from ICASE for his visit to ICASE in 1999 during which part of this work was done. LSL would like to acknowledge the support from CNRS for his visits to Laboratoire ASCI in 1998 during which part of this work was done, and the partial support from NASA LaRC under the program of Innovative Algorithms for Aerospace Engineering Analysis and Optimization. The authors would like to thank Prof. D. d'Humières of Ecole Normal Supérieure and ASCI for many enlightening discussions, and are grateful to Dr. R. Rubinstein of ICASE for his careful reading of the manuscript, to M. Salas, the Director of ICASE, for his support and encouragement of this work, and to Prof. W. Shyy and Prof. R. Mei of University of Florida for their insightful comments.

\section{Appendix A. Coupling Between Density and Other Modes.}

To consider the coupling between the density fluctuation $\delta \rho=\rho-\langle\rho\rangle$ and other modes, $e, \varepsilon, p_{x x}$, and 
$p_{x y}$, the equilibrium values of these modes are modified as the following:

$$
\begin{aligned}
& e^{(\mathrm{eq})}=\alpha_{2} \rho+\gamma_{2}\left(j_{x}^{2}+j_{y}^{2}\right)(2-\rho), \\
& \varepsilon^{(\mathrm{eq})}=\alpha_{3} \rho+\gamma_{4}\left(j_{x}^{2}+j_{y}^{2}\right)(2-\rho), \\
& p_{x x}^{(\mathrm{eq})}=\gamma_{1}\left(j_{x}^{2}+j_{y}^{2}\right)(2-\rho), \\
& p_{x y}^{(\mathrm{eq})}=\gamma_{3}\left(j_{x} j_{y}\right)(2-\rho),
\end{aligned}
$$

where $(2-\rho)$ is used to linearly approximate $1 / \rho$ when the averaged density $\rho_{0} \equiv\langle\rho\rangle=1$. With the above modifications, four elements in the first column of the linearized collision operator $C$ accordingly become:

$$
\begin{aligned}
& \mathrm{C}_{12}=s_{2}\left[\frac{1}{4} \alpha_{2}-\frac{1}{6} \gamma_{2}\left(V_{x}^{2}+V_{y}^{2}\right)\right], \\
& \mathrm{C}_{13}=s_{3}\left[\frac{1}{4} \alpha_{3}-\frac{1}{6} \gamma_{4}\left(V_{x}^{2}+V_{y}^{2}\right)\right], \\
& \mathrm{C}_{18}=-\frac{3}{2} s_{8} \gamma_{1}\left(V_{x}^{2}-V_{y}^{2}\right), \\
& \mathrm{C}_{19}=-\frac{3}{2} s_{9} \gamma_{3} V_{x} V_{y} .
\end{aligned}
$$

Based on the linearized collision operator with the above changes, the shear and the bulk viscosity at the limit of $\boldsymbol{k} \rightarrow 0$ are:

$$
\begin{aligned}
\nu_{0}= & \frac{1}{3}\left(1-V^{2} \cos ^{2} \phi\right)\left(\frac{1}{s_{8}}-\frac{1}{2}\right) \\
\eta_{0}= & \frac{1}{12 s_{2}^{2}}\left(2-3 c_{s}^{2}\right)\left(2-s_{2}\right)-\frac{V \cos \phi}{12 c_{s} s_{2} s_{8}}\left(1-3 c_{s}^{2}\right)\left(3 s_{2} s_{8}-2 s_{2}-4 s_{8}\right) \\
& +\frac{V^{2}}{4 s_{2} s_{8}}\left[s_{2}-s_{8}+2\left(s_{2} s_{8}-s_{2}-s_{8}\right) \cos ^{2} \phi\right] \\
& +\frac{V^{3} \cos \phi}{4 c_{s} s_{2} s_{8}}\left[s_{2}-s_{8}+s_{2}\left(s_{8}-2\right) \cos ^{2} \phi\right] .
\end{aligned}
$$

The sound modes propagate with velocity $V \pm c_{s}$ (at first order in $k$ ). The Galilean-coefficient up to $O\left(k^{2}\right)$ is:

$$
\begin{aligned}
g= & 1+\frac{k^{2}}{3 s_{5} s_{8}^{2}}\left[\left(s_{8}-2\right)\left(s_{5}-s_{8}\right)\left(s_{5} s_{8}-3 s_{5}-3 s_{8}+6\right)+\left(\cos ^{4} \theta-\cos ^{2} \theta\right)\right] \\
& +\frac{k^{2} V^{2}}{6 c_{s}^{2} s_{2} s_{8}^{2}}\left[\left(2-s_{8}\right)\left(s_{8}-s_{2}\right) \sin ^{2} \phi+2 c_{s}^{2} s_{2}\left(s_{8}^{2}-6 s_{8}+6\right) \cos ^{2} \phi\right]
\end{aligned}
$$

\section{Appendix B. Interpolated LBE Scheme.}

Recently, it has been proposed to use interpolation schemes to interpolate $\left\{f_{\alpha}\right\}$ from a fine mesh to a coarse mesh in order to improve the spatial resolution calculations for a limited cost in total number of nodes $[21,22]$. Obviously, the interpolation schemes create additional numerical viscosities. The Chapman-Enskog analysis shows that any second or higher order interpolation scheme does not affect the viscosities in the limit $\boldsymbol{k} \rightarrow 0$ on the fine mesh. A problem with much greater importance in practice is to calculate the viscosity at finite $\boldsymbol{k}$. To our knowledge, no such analysis is now available in the literature.

In the interpolated LBE schemes, the advection step is altered by the interpolation scheme chosen, whilst the collision step remains unchanged. The advection on a fine mesh combined with interpolation on a coarse mesh is the reconstruction step on the coarse mesh. Therefore, to obtain the modified linearized 
evolution operator $L$ only the advection operation $A$ must be changed. In what follows, we shall consider a coarse mesh with lattice constant $\delta_{x}$, and time step $\delta_{t}$. The lattice constant of a underlying fine mesh is $r \delta_{x}$, with $r \leq 1$. Effectively, the hopping velocities of particles are reduced by a factor of $r$ on coarse mesh. Therefore, dimensional analysis suggests that the sound speed is reduced by a factor of $r$, and the viscosities are reduced by a factor of $r^{2}$ in the limit $\boldsymbol{k}=\mathbf{0}$. However, the dimensional analysis does not provide any information about the quantitative effects of interpolation when $\boldsymbol{k}$ is finite. We shall analyze the effects of some commonly used second-order interpolation schemes in the LBE methods. For simplicity, we shall only deal with a uniform mesh with square grids.

B.1. Central interpolation. The reconstruction step with second-order central interpolation is given by the following formula:

$$
f_{\alpha}\left(\boldsymbol{r}_{j}\right)=\frac{r(r-1)}{2} f_{\alpha}^{*}\left(\boldsymbol{r}_{j}-\delta \boldsymbol{r}_{\alpha}\right)+\left(1-r^{2}\right) f_{\alpha}^{*}\left(\boldsymbol{r}_{j}\right)+\frac{r(r+1)}{2} f_{\alpha}^{*}\left(\boldsymbol{r}_{j}+\delta \boldsymbol{r}_{\alpha}\right),
$$

where $f_{\alpha}^{*}$ is the post-collision value of $f_{\alpha}$, i.e.,

$$
f_{\alpha}^{*} \equiv f_{\alpha}+\Omega_{\alpha}\left(f_{\alpha}\right)
$$

and

$$
\delta \boldsymbol{r}_{\alpha}=\frac{1}{r} \boldsymbol{e}_{\alpha}
$$

The advection operator in this case becomes:

$$
\mathrm{A}=\operatorname{diag}(1, A, C, B, D, A C, C B, B D, D A),
$$

where

$$
\begin{aligned}
& A=\frac{r(r+1) p}{2}+\left(1-r^{2}\right)+\frac{r(r-1)}{2 p}, \\
& B=\frac{r(r+1)}{2 p}+\left(1-r^{2}\right)+\frac{r(r-1) p}{2}, \\
& C=\frac{r(r+1) q}{2}+\left(1-r^{2}\right)+\frac{r(r-1)}{2 q}, \\
& D=\frac{r(r+1)}{2 q}+\left(1-r^{2}\right)+\frac{r(r-1) q}{2},
\end{aligned}
$$

where $p=e^{i k_{x}}$ and $q=e^{i k_{y}}$. With the new phase factors, we find new results at order 1 and 2 in $k$. The speed of sound and the "Galilean coefficient" are multiplied by $r$ and the viscosity coefficients are multiplied by $r^{2}$.

At higher order in $\boldsymbol{k}$, dispersion effects due to lattice arise, leading to differences between solutions of the standard Navier-Stokes equations and the flows computed used the LBE technique.

As in Eq. (5.24), we find that the advection coefficient for shear waves can be made isotropic to second order in $\boldsymbol{k}$ by choosing

$$
s_{5}=3 r^{2} \frac{\left(2-s_{8}\right)}{\left(3 r^{2}-s_{8}\right)}
$$

which improves Eq. (5.24), since we can choose $s_{8}$ close to 2 while maintaining $s_{5}$ reasonably far away from 2 (between 1 and $3 / 2$ ) by taking $r^{2}$ close to $2 / 3$. 
B.2. Upwind interpolation. The upwind direction in the LBE method is relative to the particle velocity $\boldsymbol{e}_{\alpha}$ (the characteristics) rather than the flow velocity $\boldsymbol{u}$. Therefore, the interpolation stencil is static in time. Second-order upwind interpolation leads to

$$
f_{\alpha}\left(\boldsymbol{r}_{j}\right)=\frac{r(r-1)}{2} f_{\alpha}^{*}\left(\boldsymbol{r}_{j}-2 \delta \boldsymbol{r}_{\alpha}\right)+r(2-r) f_{\alpha}^{*}\left(\boldsymbol{r}_{j}-\delta \boldsymbol{r}_{\alpha}\right)+\frac{(1-r)(2-r)}{2} f_{\alpha}^{*}\left(\boldsymbol{r}_{j}\right),
$$

where $\delta r_{\alpha}$ is defined in Eq. (B.3). Accordingly, the phase factors in the advection operator given by Eq. (B.4) become:

$$
\begin{aligned}
& A=\frac{(1-r)(2-r)}{2}+\frac{r(2-r)}{p}+\frac{r(r-1)}{2 p^{2}}, \\
& B=\frac{(1-r)(2-r)}{2}+r(2-r) p+\frac{r(r-1) p^{2}}{2}, \\
& C=\frac{(1-r)(2-r)}{2}+\frac{r(2-r)}{q}+\frac{r(r-1)}{2 q^{2}}, \\
& D=\frac{(1-r)(2-r)}{2}+r(2-r) q+\frac{r(r-1) q^{2}}{2},
\end{aligned}
$$

where $p=e^{i k_{x}}$ and $q=e^{i k_{y}}$.

Again, the third order term $\left(g_{1}\right)$ in $\boldsymbol{k}$ for the shear mode is anisotropic unless the following relation is satisfied:

$$
s_{5}=r^{2} \frac{\left(2-s_{8}\right)}{\left(3 r^{2}-3 r s_{8}+2 s_{8}\right)} .
$$

For $s_{8}$ and $s_{5}$ in the usual range ( $s_{8}$ near 2 and $s_{5}$ between 1 and $3 / 2$ ), the preceding equation leads to a complex value of $r$. It should be pointed out that due to the commutativity of propagation along $x$-axis and $y$-axis, one could apply different interpolation formulae along each axis, according to physics of the flow. For instance, large stretch of grid can be applied in the direction along which flow fields do not change much in space, whereas in the other orthogonal direction, a normal grid (without interpolation) or even a refined grid [9] can be used, so that the aspect ratio of the meshes is large enough to be appropriate to the flow.

Figures 7 show the $\boldsymbol{k}$-dependence of the normalized shear viscosity $\nu(\boldsymbol{k}) / \nu_{0}$ for the LBE model with and without interpolation schemes. Three orientations of $\boldsymbol{k}$ are chosen: $\theta=0$ (solid line), $\pi / 8$ (dotted line), and $\pi / 4$ (dashed line). Figs. $7(\mathrm{a}), 7(\mathrm{~b})$, and 7 (c) show the $\nu(\boldsymbol{k}) / \nu_{0}$ for the LBE model with no interpolation, with second order central interpolation scheme and $r=0.5$, and with second order upwind interpolation scheme and $r=0.5$, respectively. It should be stressed that interpolation schemes do create an enormous amount of numerical viscosity at $k=\pi / 2$ : Both the central and the upwind interpolation schemes increase the shear viscosity at $k=\pi / 2$ by almost two order of magnitude, whereas without interpolation, corresponding increase for the LBE scheme is at most only a factor of about 2.5 (in the direction $\theta=\pi / 8$ ). In all cases, the viscosity displays significant anisotropy at $k=\pi / 2$.

Similar to Figs. 7, Fig. 8 shows the $\boldsymbol{k}$-dependence of the Galilean-coefficient $g(\boldsymbol{k})$. The three curves in the middle of the figure corresponding to the LBE model without interpolation. The lower three curves, $g(k) \leq 1$, correspond to the LBE scheme with the central interpolation, and the upper three curves, $g(\boldsymbol{k}) \geq 1$, correspond to the LBE scheme with the upwind interpolation. Again, interpolations create significant effects on Galilean invariance.

One common feature observed in Figs. 7 and 8 is that the transport coefficients of a model along the direction of $\theta=\pi / 8$ is far apart from those along the directions $\theta=0$ and $\theta=\pi / 4$. This is related to the fact that for the square lattice, the wave vector $k$ along the direction $\theta=\pi / 8$ is not a reciprocal vector of the underlying lattice. 


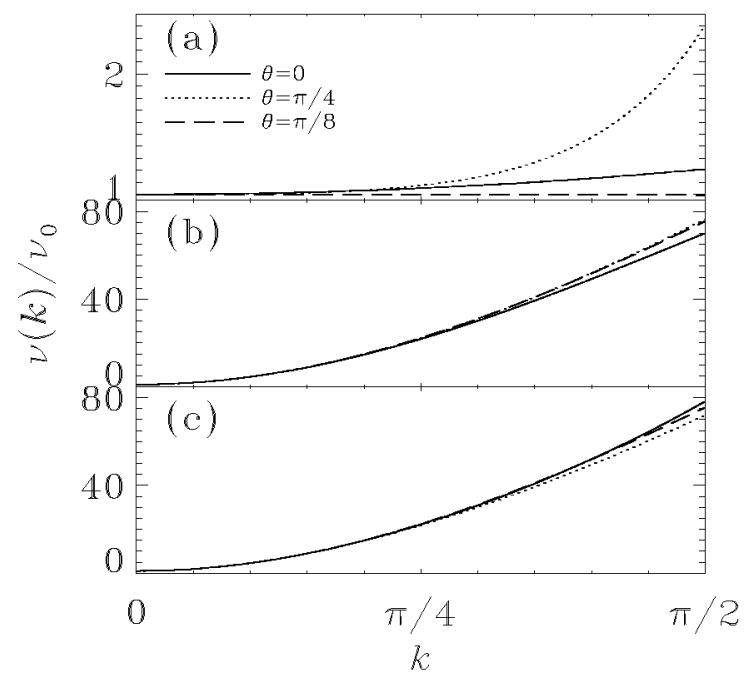

FIG. 7. The $k$-dependence of viscosities for various models. The values of the adjustable parameters and the relaxation parameters are the same as in Figs. 1 . The solid lines, dotted lines, and dashed lines correspond to $\theta=0, \pi / 8$, and $\pi / 4$, respectively. (a) The normal LBE model with no interpolation. (b) with central interpolation, and (c) with upwind interpolation.

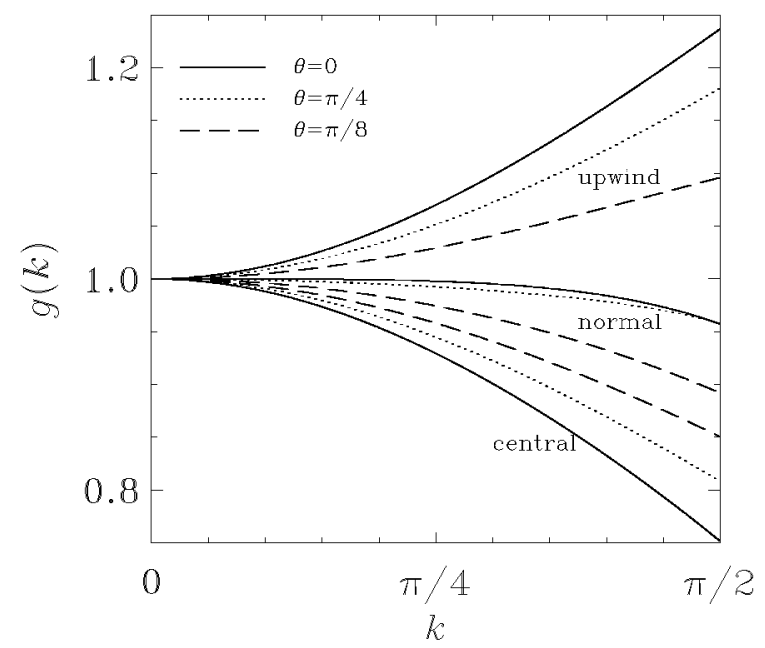

FIG. 8. The $k$-dependence of the Galilean-coefficient $g$ for various models. The solid lines, dotted lines, and dashed lines correspond to $\theta=0, \pi / 8$, and $\pi / 4$, respectively. The middle three curves are $g(k)$ for the LBE model without interpolation, the lower three for the $L B E$ model with the central interpolation and $r=0.5$, and the upper three for the LBE model with the upwind interpolation and $r=0.5$.

\section{REFERENCES}

[1] T. ABE, Derivation of the lattice Boltzmann method by means of the discrete ordinate method for the Boltzmann equation, J. Comp. Phys., 131 (1997), pp. 241-246.

[2] O. Behrend, R. Harris, and P.B. Warren, Hydrodynamic behavior of lattice Boltzmann and lattice Bhatnagar-Gross-Krook models, Phys. Rev. E, 50 (1994), pp. 4586-4595.

[3] R. Benzi, S. Succi, and M. Vergassola, The lattice Boltzmann equation: Theory and applications, Phys. Rep., 222 (1992), pp. 145-197.

[4] P.L. Bhatnagar, E.P. Gross, AND M. Krook, A model for collision processes in gases. I. Small amplitude processes in charged and neutral one-component systems, Phys. Rev., 94 (1954), pp. 511- 
525.

[5] H. Chen, S. Chen, and W.H. Matthaeus, Recovery of the Navier-Stokes equations using a lattice gas Boltzmann method, Phys. Rev. A, 45 (1992), pp. R5339-R5342.

[6] S. Chen And G.D. Doolen, Lattice Boltzmann method for fluid flows, Annu. Rev. Fluid Mech., 30 (1998), pp. 329-364.

[7] S.P. Das, H.J. Bussemaker, And M.H. ERnst, Generalized hydrodynamics and dispersion-relations in lattice gases, Phys. Rev. E, 48 (1993), pp. 245-255.

[8] D. D'Humières, Generalized lattice-Boltzmann equations, in Rarefied Gas Dynamics: Theory and Simulations, Prog. Astro. Aeor., Vol. 159, edited by B. D. Shizgal and D. P. Weaver, AIAA, Washington, DC, 1992.

[9] O. Filippova And D. HÄNEL, Grid refinement for lattice-BGK models, J. Comp. Phys., 147 (1998), pp. 219-228.

[10] U. Frisch, B. Hasslacher, And Y. Pomeau, Lattice-gas automata for the Navier-Stokes equation, Phys. Rev. Lett., 56 (1986), pp. 1505-1508.

[11] U. Frisch, D. D'Humières, B. Hasslacher, P. Lallemand, Y. Pomeau, and J.-P. Rivet, Lattice gas hydrodynamics in two and three dimensions, Complex Systems, 1 (1987), pp. 649-707.

[12] L. Giraud, Ph.D. Thesis, Université Paris VI, 1997.

[13] L. Giraud, D. D'Humières, and P. Lallemand, A lattice Boltzmann model for Jeffreys viscoelastic fluid, Europhys. Lett., 42 (1998), pp. 625-630.

[14] P. Grosfils, J.-P. Boon, R. Brito, And M.H. Ernst, Statistical hydrodynamics of lattice-gas automata, Phys. Rev. E, 48 (1993), 2655-2668.

[15] P. Grosfils and P. Lallemand, Dispersion effects in lattice gases with internal and translational mode-coupling, Europhys. Lett., 24 (1993), pp. 473-478.

[16] X. HE AND G. Doolen, Lattice Boltzmann method on curvilinear coordinates system: Flow around a circular cylinder, J. Comp. Phys., 134 (1997), pp. 306-315.

$[17] \longrightarrow$ Lattice Boltzmann method on a curvilinear coordinate system: Vortex shedding behind a circularcylinder, Phys. Rev. E, 56 (1997), pp. 434-440.

[18] X. HE AND L.-S. Luo, Lattice Boltzmann model for the incompressible Navier-Stokes equation, J. Stat. Phys., 88 (1997), pp. 927-944.

[19] — A priori derivation of the lattice Boltzmann equation, Phys. Rev. E, 55 (1997), pp. R6333-R6336.

[20] — Theory of the lattice Boltzmann method: From the Boltzmann equation to the lattice Boltzmann equation, Phys. Rev. E, 56 (1997), pp. 6811-6817.

[21] X. He, L.-S. Luo, And M. Dembo, Some progress in lattice Boltzmann method. Part I. Nonuniform mesh grids, J. Comp. Phys., 129 (1996), pp. 357-363.

[22] _ Some progress in lattice Boltzmann method. Reynolds number enhancement in simulations, Physica A, 239 (1997), pp. 276-285.

[23] X. He, X. Shan, ANd G.D. Doolen, Discrete Boltzmann-equation model for nonideal gases, Phys. Rev. E, 57 (1998), pp. R13-R16.

[24] X. HE, S. Chen, AND R. ZHANG, A lattice Boltzmann scheme for incompressible multiphase flow and its application in simulation of Rayleigh-Taylor instability, J. Comput. Phys., 152 (1999), pp. 642-663.

[25] X. He, R. Zhang, S. Chen, And G.D. Doolen, On the three-dimensional Rayleigh-Taylor instability, Phys. Fluids, 11 (1999), pp. 1143-1152. 
[26] J.O. Hirschfelder, C.F. Curtiss, and R.B. Bird, Molecular Theory of Gases and Liquids, Wiley, New York, 1954.

[27] S. Hou, Q. Zou, S. Chen, G.D. Doolen, And A.C. Cogley, Simulation of cavity flow by the lattice Boltzmann method, J. Comput. Phys., 118 (1995), pp. 329-347.

[28] M. JunK, Kinetic schemes in the case of low Mach numbers, J. Comput. Phys., 151 (1999), pp. 947-968.

[29] L.D. Landau and E.M. Lifshitz, Fluid Mechanics, 2nd ed., Pergamon, Oxford, 1987.

[30] L.-S. Luo, Lattice-gas automoata and lattice Boltzmann equations for two-dimensional hydrodynamics, Ph.D. Thesis, Georgia Institute of Technology, 1993.

[31] — Symmetry-breaking of flow in 2D symmetrical channels: Simulations by lattice-Boltzmann method, Int. J. Mod. Phys. C, 8 (1997), pp. 859-867.

[32] — Unified theory of the lattice Boltzmann models for non-ideal gases, Phys. Rev. Lett., 81 (1998), pp. 1618-1621.

[33] L.-S. Luo, H. Chen, S. Chen, G.D. Doolen, And Y.-C. Lee, Generalized hydrodynamic transport in lattice-gas automata, Phys. Rev. A, 43 (1991), pp. 7097-7080.

[34] G. MCNamara AND G. ZANetTi, Use of the Boltzmann equation to simulate lattice-gas automata, Phys. Rev. Lett., 61 (1988), pp. 2332-2335.

[35] D.W. QI, Lattice-Boltzmann simulations of particles in non-zero-Reynolds-number flows, J. Fluid Mech., 385 (1999), pp. 41-62.

[36] Y.H. QIAN, D. D'Humières, And P. Lallemand, Lattice BGK models for Navier-Stokes equation, Europhys. Lett., 17 (1992), pp. 479-484.

[37] Y.H. QIAN AND Y. Zhou, Complete Galilean-invariant lattice BGK models for the Navier-Stokes equation, Europhys. Lett., 42 (1998), pp. 359-364.

[38] Y.H. QTAN AND Y. ZHOU, High-order dynamics in lattice-based models using the Chapman-Enskog method, Phys. Rev. E, 61 (2000), pp. 2103-2106. [ICASE Report No. 99-21 (1999)].

[39] Y. SAAD, Numerical method for large eigenvalue problems, Manchester University Press, Manchester, 1993.

[40] X. Shan And H. Chen, Lattice Boltzmann model for simulating flows with multiple phases and components, Phys. Rev. E, 47 (1993), pp. 1815-1819.

[41] _ Simulation of nonideal gases and liquid-gas phase-transitions by the lattice Boltzmann-equation, Phys. Rev. E, 49 (1994), pp. 2941-2948.

[42] J.D. Sterling And S. Chen, Stability analysis of lattice Boltzmann methods, J. Comput. Phys., 123 (1995), pp. 196-206.

[43] M.R. Swift, E. Orlandini, W.R. Osborn, and J.M. Yeomans, Lattice Boltzmann simulations of liquid-gas and binary-fluid systems, Phys. Rev. E, 54 (1996), pp. 5041-5052.

[44] M.R. Swift, W.R. Osborn, And J.M. Yeomans, Lattice Boltzmann simulation of nonideal fluids, Phys. Rev. Lett., 75 (1995), pp. 830-833.

[45] F. Toschi, G. Amati, S. Succi, R. Benzi, And R. Piva, Intermittency and structure function in channel flow turbulence, Phys. Rev. Lett., 82 (1999), pp. 5044-5047.

[46] S. Wolfram, Cellular automaton fluids 1. Basic theory, J. Stat. Phys., 45 (1986), pp. 471-526.

[47] R.A. Worthing, J. Mozer, And G. Seetey, Stability of lattice Boltzmann methods in hydrodynamic regimes, Phys. Rev. E, 56 (1997), pp. 2246-2253. 


\begin{tabular}{|c|c|c|c|}
\hline \multicolumn{3}{|c|}{ REPORT DOCUMENTATION PAGE } & $\begin{array}{l}\text { Form Approved } \\
\text { OMB No. 0704-0188 }\end{array}$ \\
\hline \multicolumn{4}{|c|}{ 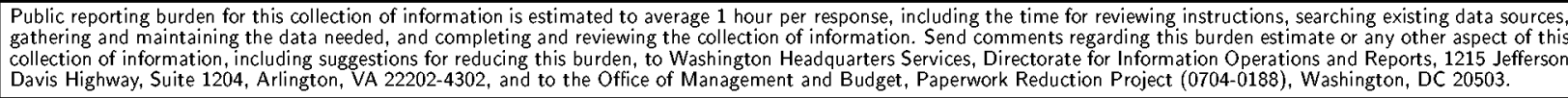 } \\
\hline 1. AGENCY USE ONLY(Leave blank) & $\begin{array}{l}\text { 2. REPORT DATE } \\
\text { April } 2000\end{array}$ & \multicolumn{2}{|c|}{$\begin{array}{l}\text { 3. REPORT TYPE AND DATES COVERED } \\
\text { Contractor Report }\end{array}$} \\
\hline \multicolumn{3}{|c|}{$\begin{array}{l}\text { 4. TITLE AND SUBTITLE } \\
\text { Theory of the lattice Boltzmann method: Dispersion, dissipation, } \\
\text { isotropy, Galilean invariance, and stability }\end{array}$} & \multirow[t]{2}{*}{$\begin{array}{l}\text { 5. FUNDING NUMBERS } \\
\text { C NAS1-97046 } \\
\text { WU } 505-90-52-01\end{array}$} \\
\hline \multicolumn{3}{|c|}{$\begin{array}{l}\text { 6. AUTHOR(S) } \\
\text { Pierre Lallemand and Li-Shi Luo }\end{array}$} & \\
\hline \multicolumn{3}{|c|}{$\begin{array}{l}\text { 7. PERFORMING ORGANIZATION NAME(S) AND ADDRESS(ES) } \\
\text { Institute for Computer Applications in Science and Engineering } \\
\text { Mail Stop 132C, NASA Langley Research Center } \\
\text { Hampton, VA 23681-2199 }\end{array}$} & $\begin{array}{l}\text { 8. PERFORMING ORGANIZATION } \\
\text { REPORT NUMBER } \\
\text { ICASE Report No. } 2000-17\end{array}$ \\
\hline \multicolumn{2}{|c|}{$\begin{array}{l}\text { 9. SPONSORING/MONITORING AGENCY NAME(S) AND ADDRESS(ES } \\
\text { National Aeronautics and Space Administration } \\
\text { Langley Research Center } \\
\text { Hampton, VA 23681-2199 }\end{array}$} & $\begin{array}{l}\text { 10. SPONS } \\
\text { AGENC } \\
\text { NASA } \\
\text { ICASI }\end{array}$ & $\begin{array}{l}\text { 10. SPONSORING/MONITORING } \\
\text { AGENCY REPORT NUMBER } \\
\text { NASA/CR-2000-210103 } \\
\text { ICASE Report No. } 2000-17\end{array}$ \\
\hline \multicolumn{4}{|c|}{$\begin{array}{l}\text { 11. SUPPLEMENTARY NOTES } \\
\text { Langley Technical Monitor: Dennis M. Bushnell } \\
\text { Final Report } \\
\text { To appear in Physical Review E. }\end{array}$} \\
\hline \multicolumn{2}{|c|}{$\begin{array}{l}\text { 12a. DISTRIBUTION/AVAILABILITY STATEMENT } \\
\text { Unclassified-Unlimited } \\
\text { Subject Category } 34 \\
\text { Distribution: Nonstandard } \\
\text { Availability: NASA-CASI (301) 621-0390 }\end{array}$} & & 12b. DISTRIBUTION CODE \\
\hline \multicolumn{4}{|c|}{$\begin{array}{l}\text { 13. ABSTRACT (Maximum } 200 \text { words) } \\
\text { The generalized hydrodynamics (the wave vector dependence of the transport coefficients) of a generalized lattice } \\
\text { Boltzmann equation (LBE) is studied in detail. The generalized lattice Boltzmann equation is constructed in moment } \\
\text { space rather than in discrete velocity space. The generalized hydrodynamics of the model is obtained by solving } \\
\text { the dispersion equation of the linearized LBE either analytically by using perturbation technique or numerically. } \\
\text { The proposed LBE model has a maximum number of adjustable parameters for the given set of discrete velocities. } \\
\text { Generalized hydrodynamics characterizes dispersion, dissipation (hyper-viscosities), anisotropy, and lack of Galilean } \\
\text { invariance of the model, and can be applied to select the values of the adjustable parameters which optimize the } \\
\text { properties of the model. The proposed generalized hydrodynamic analysis also provides some insights into stability } \\
\text { and proper initial conditions for LBE simulations. The stability properties of some 2D LBE models are analyzed and } \\
\text { compared with each other in the parameter space of the mean streaming velocity and the viscous relaxation time. } \\
\text { The procedure described in this work can be applied to analyze other LBE models. As examples, LBE models with } \\
\text { various interpolation schemes are analyzed. Numerical results on shear flow with an initially discontinuous velocity } \\
\text { profile (shock) with or without a constant streaming velocity are shown to demonstrate the dispersion effects in } \\
\text { the LBE model; the results compare favorably with our theoretical analysis. We also show that whereas linear } \\
\text { analysis of the LBE evolution operator is equivalent to Chapman-Enskog analysis in the long wave-length limit } \\
\text { (wave vector k=0), it can also provide results for large values of } \mathbf{k} \text {. Such results are important for the stability and } \\
\text { other hydrodynamic properties of the LBE method and cannot be obtained through Chapman-Enskog analysis. }\end{array}$} \\
\hline \multirow{2}{*}{\multicolumn{3}{|c|}{$\begin{array}{l}\text { 14. SUBJECT TERMS } \\
\text { kinetic method, lattice Boltzmann equation, derivation of hydrodynamic equation, } \\
\text { stability analysis, numerical artifacts of the LBE method }\end{array}$}} & $\begin{array}{l}\text { 15. NUMBER OF PAGES } \\
33 \\
\end{array}$ \\
\hline & & & $\begin{array}{c}\text { 16. PRICE CODE } \\
\text { A03 }\end{array}$ \\
\hline $\begin{array}{l}\text { 17. SECURITY CLASSIFICATION } \\
\text { OF REPORT } \\
\text { Unclassified }\end{array}$ & $\begin{array}{l}\text { 18. SECURITY CLASSIFICATION } \\
\text { OF THIS PAGE } \\
\text { Unclassified }\end{array}$ & $\begin{array}{l}\text { 19. SECURITY CLASSIFICATION } \\
\text { OF ABSTRACT }\end{array}$ & $\begin{array}{l}\text { 20. LIMITATION } \\
\text { OF ABSTRACT }\end{array}$ \\
\hline NSN 7540-01-280-5500 & & & $\begin{array}{l}\text { Standard Form 298(Rev. } 2-89) \\
\text { Prescribed by ANSI Std. Z39-18 } \\
\text { 298-102 }\end{array}$ \\
\hline
\end{tabular}

